ESAIM: COCV $22(2016) 112-133$

DOI: $10.1051 / \mathrm{cocv} / 2014068$
ESAIM: Control, Optimisation and Calculus of Variations

www.esaim-cocv.org

\title{
DEV-DIV- AND DEVSYM-DEVCURL-INEQUALITIES FOR INCOMPATIBLE SQUARE TENSOR FIELDS WITH MIXED BOUNDARY CONDITIONS
}

\author{
Sebastian Bauer ${ }^{1}$, Patrizio NefF ${ }^{1}$, Dirk Pauly ${ }^{1}$ and Gerhard Starke ${ }^{1}$
}

\begin{abstract}
Let $\Omega \subset \mathbb{R}^{n}, n \geq 2$, be a bounded Lipschitz domain and $1<q<\infty$. We prove the inequality

$$
\|T\|_{L^{q}(\Omega)} \leq C_{D D}\left(\|\operatorname{dev} T\|_{L^{q}(\Omega)}+\|\operatorname{Div} T\|_{L^{q}(\Omega)}\right)
$$

being valid for tensor fields $T: \Omega \rightarrow \mathbb{R}^{n \times n}$ with a normal boundary condition on some open and non-empty part $\Gamma_{\nu}$ of the boundary $\partial \Omega$. Here $\operatorname{dev} T=T-\frac{1}{n} \operatorname{tr}(T) \cdot$ Id denotes the deviatoric part of the tensor $T$ and Div is the divergence row-wise. Furthermore, we prove

$$
\begin{aligned}
& \|T\|_{L^{2}(\Omega)} \leq C_{D S C}\left(\|\operatorname{dev} \operatorname{sym} T\|_{L^{2}(\Omega)}+\|\operatorname{Curl} T\|_{L^{2}(\Omega)}\right) \quad \text { if } n \geq 3, \\
& \|T\|_{L^{2}(\Omega)} \leq C_{D S D C}\left(\|\operatorname{dev} \operatorname{sym} T\|_{L^{2}(\Omega)}+\|\operatorname{dev} \operatorname{Curl} T\|_{L^{2}(\Omega)}\right) \quad \text { if } n=3,
\end{aligned}
$$

being valid for tensor fields $T$ with a tangential boundary condition on some open and non-empty part $\Gamma_{\tau}$ of $\partial \Omega$. Here, $\operatorname{sym} T=\frac{1}{2}\left(T+T^{\top}\right)$ denotes the symmetric part of $T$ and Curl is the rotation row-wise.
\end{abstract}

Mathematics Subject Classification. 35A23, 35Q61, 74C05, 78A25, 78A30.

Received February 5, 2014. Revised July 29, 2014.

Published online November 23, 2015.

\section{INTRODUCTION}

'Every mathematical theorem has an inequality behind it ...' In this work we consider $(n \times n)$-tensor fields $T$ on bounded domains $\Omega \subset \mathbb{R}^{n}, n \geq 2$, with Lipschitz-continuous boundary $\partial \Omega$. Such a tensor field may be decomposed pointwise orthogonally in its symmetric part and its skew-symmetric part

$$
T=\operatorname{sym} T+\operatorname{skew} T,
$$

where sym $T=\frac{1}{2}\left(T+T^{\top}\right)$ and skew $T=\frac{1}{2}\left(T-T^{\top}\right)$. In the recent paper [29], it has been shown that in $L^{2}(\Omega)$ the skew symmetric part of $T$ is controlled by the symmetric part and the Curl of $T$, leading to

$$
\|T\|_{L^{2}(\Omega)} \leq C_{S C}\left(\|\operatorname{sym} T\|_{L^{2}(\Omega)}+\|\operatorname{Curl} T\|_{L^{2}(\Omega)}\right),
$$

Keywords and phrases. Korn's inequality, Lie-algebra decomposition, Poincaré's inequality, Maxwell estimates, relaxed micromorphic model.

1 Fakultät für Mathematik, Universität Duisburg-Essen, Campus Essen, Thea-Leymann-Str. 9, 45127 Essen, Germany. sebastian.bauer.seuberlich@uni-due.de 
if a tangential boundary condition is imposed on some non-empty and open part $\Gamma_{\tau}$ of the boundary $\partial \Omega$. In classical terms $\left.T \tau\right|_{\Gamma_{\tau}}=0$ is needed for all tangential-vectors $\tau$ on $\Gamma_{\tau}$. Here and hereafter all differential operators on tensor fields are taken row-wise. For exact definitions of operators and function spaces, see Section 2. We shall call this inequality the Sym-Curl-inequality. Since the Curl operator vanishes on gradients, a certain variant of Korn's first inequality follows immediately, i.e., with $T=\operatorname{Grad} v$ and Curl Grad $=0$ we have

$$
\|\operatorname{Grad} v\|_{L^{2}(\Omega)} \leq C_{S C}\|\operatorname{sym} \operatorname{Grad} v\|_{L^{2}(\Omega)}
$$

for all $v \in H^{1}(\Omega)$ with $\left.(\operatorname{Grad} v) \tau\right|_{\Gamma_{\tau}}=0$. Obviously, this boundary condition is a weakening of the usual Dirichlet boundary condition $\left.v\right|_{\Gamma_{\tau}}=0$, see the discussion in [27].

The tensor $T$ may also be decomposed pointwise orthogonally in its trace-free or deviatoric and its trace or spherical part

$$
T=\operatorname{dev} T+\frac{1}{n} \operatorname{tr}(T) \cdot \mathrm{Id},
$$

where Id denotes the identity matrix in $\mathbb{R}^{n}$ and $\operatorname{tr} T=\sum_{i=1}^{n} T_{i i}$.

In Theorem 3.1 of this contribution, we show that in $L^{q}(\Omega), 1<q<\infty$, the trace part of $T$ is controlled by the deviatoric part and the divergence of $T$, i.e.,

$$
\|T\|_{L^{q}(\Omega)} \leq C_{D D}\left(\|\operatorname{dev} T\|_{L^{q}(\Omega)}+\|\operatorname{Div} T\|_{L^{q}(\Omega)}\right),
$$

if a normal boundary condition is imposed on some non-empty and open part $\Gamma_{\nu}$ of $\partial \Omega$. In classical terms

$$
\left.T \nu\right|_{\Gamma_{\nu}}=0
$$

is needed for the normal vector $\nu$ at $\Gamma_{\nu}$. We shall call this inequality the Dev-Div-inequality.

In case that $n=3$ and $T=\operatorname{Curl} S$ is already a Curl of a tensor field $S$ having the proper tangential boundary condition on $\Gamma_{\tau}$, we conclude that $T$ is already controlled by its deviatoric part alone, i.e.,

$$
\|\operatorname{Curl} S\|_{L^{q}(\Omega)} \leq C_{D D}\|\operatorname{dev} \operatorname{Curl} S\|_{L^{q}(\Omega)},
$$

since Div Curl $=0$ and $T$ inherits the proper normal boundary condition from $S$. The inequality (1.6) may be seen as a Korn-type inequality, cf. (1.3). Both orthogonal decompositions (1.1) and (1.4) may be combined by appealing to the Cartan-decomposition of the Lie-algebra $\mathfrak{g l}(n)$

$$
\begin{aligned}
\mathfrak{g l}(n) & =(\mathfrak{s l}(n) \cap \operatorname{Sym}(n)) \oplus \mathfrak{s o}(n) \oplus \mathbb{R} \cdot \mathrm{Id} \\
T & =\operatorname{dev} \operatorname{sym} T \quad+\text { skew } T+\frac{1}{n} \operatorname{tr}(T) \cdot \mathrm{Id} .
\end{aligned}
$$

Here, $\mathfrak{s l}(n)$ denotes the Lie-algebra of trace free matrices and $\mathfrak{s o}(n)$ denotes the Lie-algebra of skew-symmetric matrices in $\mathbb{R}^{n \times n}$. Now, in a naive manner an estimate of the following kind could be guessed

$$
\|T\|_{L^{2}(\Omega)} \leq C\left(\|\operatorname{dev} \operatorname{sym} T\|_{L^{2}(\Omega)}+\|\operatorname{Curl} T\|_{L^{2}(\Omega)}+\|\operatorname{Div} T\|_{L^{2}(\Omega)}\right),
$$

accompanied by suitable boundary conditions. In fact, in Theorem 5.1 we prove a somewhat stronger result: For $n=3$ we prove the new DevSym-DevCurl-inequality

$$
\|T\|_{L^{2}(\Omega)} \leq C_{D S D C}\left(\|\operatorname{dev} \operatorname{sym} T\|_{L^{2}(\Omega)}+\|\operatorname{dev} \operatorname{Curl} T\|_{L^{2}(\Omega)}\right),
$$

where again a tangential boundary condition is imposed on some non-empty and open part $\Gamma_{\tau}$ of the boundary. Since the deviatoric part is only defined for quadratic tensors, this estimate does not make sense for $n \neq 3$. In general, $\operatorname{Curl} T$ is a $(n(n-1) / 2 \times n)$-matrix and we prove that for $n \geq 3$

$$
\|T\|_{L^{2}(\Omega)} \leq C_{D S C}\left(\|\operatorname{dev} \operatorname{sym} T\|_{L^{2}(\Omega)}+\|\operatorname{Curl} T\|_{L^{2}(\Omega)}\right)
$$


holds. In order to show (1.7) we first prove for $n \geq 3$ a Korn type inequality, i.e.

$$
\|\operatorname{Grad} v\|_{L^{2}(\Omega)} \leq C\|\operatorname{dev} \operatorname{sym} \operatorname{Grad} v\|_{L^{2}(\Omega)}
$$

for all $v \in H^{1}(\Omega)$ with $\left.(\operatorname{Grad} v) \tau\right|_{\Gamma_{\tau}}=0$.

Whereas inequalities of Sym-Curl-type are investigated by some of the present authors in a series of papers for the first time, see $[27,29]$, there are already several contributions to Div-Dev-type inequalities in the literature: In ([2], Lem. 3.1) a Div-Dev-estimate is proved for $n=2$ replacing the boundary condition by the average condition $\int_{\Omega} \operatorname{tr}(T) \mathrm{d} x=0$. In the proof of Theorem 3.1 we adopt the idea of proof from this Lemma. The result is extended to more general boundary conditions in [9] using a different argument. In ([4], Lem. 3.2) for $n=2$ and $n=3$ the estimate

$$
\|T\|_{L^{2}(\Omega)}^{2} \leq C\left(\frac{1}{2 \mu}\|\operatorname{dev} T\|_{L^{2}(\Omega)}^{2}+\frac{1}{n(n \lambda+2 \mu)}\|\operatorname{tr} T\|_{L^{2}(\Omega)}^{2}+\|\operatorname{Div} T\|_{H^{-1}\left(\Gamma_{\tau} ; \Omega\right)}^{2}\right)
$$

is shown by means of a Helmholtz decomposition. In the notation used in this paper $H^{-1}\left(\Gamma_{\tau} ; \Omega\right)$ denotes the dual space of $H\left(\operatorname{Grad} ; \Gamma_{\tau} ; \Omega\right)$. This estimate holds uniformly in $0<\mu_{1} \leq \mu \leq \mu_{2}$ and $0<\lambda<\infty$. Therefore, in the (incompressible) limit $\lambda \rightarrow \infty$ this estimate implies a Dev-Div-estimate. All of these contributions were derived with the application to finite element approaches of mixed type to incompressible linear elasticity in mind (cf. [3], Chap. 9) where the Dev-Div-estimate is crucial for establishing well-posedness of the variational formulation. Other applications include pseudostress-velocity formulations of Stokes flow (cf. [7], Sect. 3.2) and generalizations of it (see Sect. 7 for more details).

Korn-type estimates, replacing the symmetric gradient by its trace-free part are given in [11], i.e.

$$
\|\operatorname{Grad} v\|_{L^{2}(\Omega)} \leq C\left(\|\operatorname{dev} \operatorname{sym} \operatorname{Grad} v\|_{L^{2}(\Omega)}+\|v\|_{L^{2}(\Omega)}\right)
$$

for all $v \in H(\operatorname{Grad} ; \Omega)$ and $n \geq 3$. In ([31], Thm. 3.2) a trace-free version of Korn's first inequality is shown by means of integral representations. In detail it is shown that for $1<q<\infty$ and any projector $\Pi$ from $W^{q}(\mathrm{Grad} ; \Omega)$ onto the finite dimensional kernel of dev sym Grad, there exists a constant $C>0$, such that for all $u \in W^{q}(\mathrm{Grad} ; \Omega)$

$$
\|u-\Pi u\|_{W^{q}(\operatorname{Grad} ; \Omega)} \leq C\|\operatorname{dev} \operatorname{sym} \operatorname{Grad} u\|_{L^{q}(\Omega)} .
$$

It is well known, that for $n=2$ estimate (1.9) fails to hold true, since in this case the kernel of dev sym Grad is given by the holomorphic functions and thus is infinite-dimensional. On the other hand, in ([29], Appendix) inequality (1.8) is proved for $v \in H(\mathrm{Grad} ; \partial \Omega ; \Omega)$ by simple partial integration and some elementary estimates. In [13] it is proved that

$$
\|\operatorname{Grad} v\|_{L^{q}(\Omega)} \leq C\|\operatorname{dev} \operatorname{sym} \operatorname{Grad} v\|_{L^{q}(\Omega)}
$$

holds for $v \in W^{q}(\operatorname{Grad} ; \partial \Omega ; \Omega)$ for $n=2$ and $1<q<\infty$, and in [14] this inequality is proved for $q=1$, $v \in W^{1}(\operatorname{Grad} ; \partial \Omega ; \Omega)$ and arbitrary space dimensions $n$. In Section 6 we show that for the case of only a partial boundary condition, i.e. $v \in H\left(\operatorname{Grad} ; \Gamma_{\tau} ; \Omega\right)$, the estimate $(1.8)$ is false by means of a construction taken from [30].

What about inequalities like DevSym-DevSymCurl or other combinations? In Section 6 we give some negative results in that direction. It may be quite illuminating to see by some simple arguments, why the kernel of the operators defining the right hand side of our inequalities are trivial on, say, the space of smooth compactly supported tensor fields. Some calculations in that direction are also presented in Section 6. In Section 7 applications of the derived inequalities are given. The remaining part of the paper is organized as follows: in Section 2 we shall give notations and definitions used in this paper. In Section 3 we provide the proof of the Dev-Div-inequality and in Sections 4 and 5 we give the proofs of the DevSym-Curl- and the DevSym-DevCurl-inequality. In the Appendix we prove a representation formula for the kernel of dev sym Grad in arbitrary space dimensions used in the proof of Theorem 5.1. 


\section{DeFinitions AND PRELIMINARIES}

Throughout the entire paper we assume $\Omega \subset \mathbb{R}^{n}, n \geq 2$, to be a bounded domain with boundary $\partial \Omega$. Moreover, let $\Gamma_{\tau}$ be a relatively open subset of $\partial \Omega$ and $\Gamma_{\nu}:=\partial \Omega \backslash \bar{\Gamma}_{\tau}$. Here, the subscripts $\tau$ and $\nu$ refer to the tangential and normal boundary condition, respectively.

The usual Lebesgue-spaces of $q$-integrable functions, vector fields and tensor fields on $\Omega$ with values in $\mathbb{R}, \mathbb{R}^{n}$ and $\mathbb{R}^{n \times n}$, respectively, will be denoted by $L^{q}(\Omega)$. Moreover, we introduce the standard Sobolev-spaces

$$
\begin{aligned}
W^{q}(\operatorname{grad} ; \Omega) & :=\left\{u \in L^{q}(\Omega) \mid \operatorname{grad} u \in L^{q}(\Omega)\right\}=W^{1, q}(\Omega), \\
W^{q}(\operatorname{div} ; \Omega) & :=\left\{u \in L^{q}(\Omega) \mid \operatorname{div} u \in L^{q}(\Omega)\right\}, \\
W^{q}(\operatorname{curl} ; \Omega) & :=\left\{u \in L^{q}(\Omega) \mid \operatorname{curl} u \in L^{q}(\Omega)\right\},
\end{aligned}
$$

where grad, div and curl are the usual differential operators gradient, divergence and rotation ${ }^{2}$, respectively. All derivatives are understood in the distributional sense. For $q=2$ we replace as usual $W^{2}$ by $H$.

In order to realize certain boundary conditions we make use of the spaces

$$
C^{\infty}(\Gamma, \bar{\Omega}):=\left\{\left.u\right|_{\Omega} \mid u \in C_{0}^{\infty}\left(\mathbb{R}^{n} \backslash \bar{\Gamma}\right)\right\}
$$

for $\Gamma=\partial \Omega, \Gamma_{\tau}$ or $\Gamma_{\nu}$ and define

$$
W^{q}\left(\operatorname{grad} ; \Gamma_{\tau} ; \Omega\right), \quad W^{q}\left(\operatorname{div} ; \Gamma_{\nu} ; \Omega\right) \quad \text { and } \quad W^{q}\left(\operatorname{curl} ; \Gamma_{\tau} ; \Omega\right)
$$

as completion under the respective graph norms of the scalar-valued space $C^{\infty}\left(\Gamma_{\tau}, \bar{\Omega}\right)$ and the vector-valued spaces $C^{\infty}\left(\Gamma_{\nu}, \bar{\Omega}\right)$ and $C^{\infty}\left(\Gamma_{\tau}, \bar{\Omega}\right)$, respectively. Therefore, these spaces generalize the homogeneous Dirichlet boundary conditions

$$
\left.u\right|_{\Gamma_{\tau}}=0 \text { (scalar), }\left.\quad \nu \cdot v\right|_{\Gamma_{\nu}}=0 \text { (normal) } \quad \text { and } \quad \nu \times\left. v\right|_{\Gamma_{\tau}}=0 \text { (tangential) }
$$

respectively.

Now we extend our notations to vector and tensor fields by defining all differential operations on rows. Thus, for a vector field $v=\left(v_{1}, \ldots, v_{n}\right)^{\top}$ we define the tensor field $\operatorname{Grad} v:=\left(\operatorname{grad}^{\top} v_{1}, \ldots, \operatorname{grad}^{\top} v_{n}\right)^{\top}$, where ${ }^{\top}$ denotes the transpose. Note, that Grad $v$ is just the Jacobian of $v$. For a tensor field $T$ we define the divergence $\operatorname{Div} T:=\left(\operatorname{div} T_{1}^{\top}, \ldots, \operatorname{div} T_{n}^{\top}\right)^{\top}$ and the rotation $\operatorname{Curl} T=\left(\operatorname{curl}^{\top} T_{1}^{\top}, \ldots, \operatorname{curl}^{\top} T_{n}^{\top}\right)^{\top}$, where $T_{i}$ denote the row-vectors of $T$, i.e., $T=\left(T_{1}, \ldots, T_{n}\right)^{\top}$. The corresponding Sobolev-spaces will be denoted by

$$
W^{q}(\operatorname{Grad} ; \Omega), \quad H(\operatorname{Grad} ; \Omega), \quad W^{q}\left(\operatorname{Grad} ; \Gamma_{\tau} ; \Omega\right), \quad H\left(\operatorname{Grad} ; \Gamma_{\tau} ; \Omega\right)
$$

and so on. Note that the spaces $W^{q}\left(\operatorname{Div} ; \Gamma_{\nu} ; \Omega\right)$ and $H\left(\operatorname{Div} ; \Gamma_{\nu} ; \Omega\right)$ generalize the normal boundary condition $\left.T \nu\right|_{\Gamma_{\nu}}=0$, while the spaces $W^{q}\left(\mathrm{Curl} ; \Gamma_{\tau} ; \Omega\right)$ and $H\left(\mathrm{Curl} ; \Gamma_{\tau} ; \Omega\right)$ generalize the tangential boundary condition $\left.T \tau\right|_{\Gamma_{\tau}}=0$.

Furthermore, we define $W^{-1, q}\left(\operatorname{grad} ; \Gamma_{\nu} ; \Omega\right)$ to be the dual of $W^{p}\left(\operatorname{grad} ; \Gamma_{\tau} ; \Omega\right)$, i.e.,

$$
W^{-1, q}\left(\operatorname{grad} ; \Gamma_{\nu} ; \Omega\right):=\left(W^{p}\left(\operatorname{grad} ; \Gamma_{\tau} ; \Omega\right)\right)^{\prime},
$$

where as usual $p$ satisfies $1 / q+1 / p=1$. If $\Gamma_{\nu}=\emptyset$, i.e., $\Gamma_{\tau}=\partial \Omega$, we simply write

$$
W^{-1, q}(\operatorname{grad} ; \Omega):=\left(W^{p}(\operatorname{grad} ; \partial \Omega ; \Omega)\right)^{\prime} .
$$

Analogously we define

$$
\begin{aligned}
W^{-1, q}\left(\operatorname{Grad} ; \Gamma_{\nu} ; \Omega\right) & :=\left(W^{p}\left(\operatorname{Grad} ; \Gamma_{\tau} ; \Omega\right)\right)^{\prime}, \\
W^{-1, q}(\operatorname{Grad} ; \Omega) & :=\left(W^{p}(\operatorname{Grad} ; \partial \Omega ; \Omega)\right)^{\prime} .
\end{aligned}
$$

\footnotetext{
${ }^{2}$ For a definition of the rotation for $n \neq 3$, see, e.g. [26].
} 
In general, we only assume fairly weak regularity assumptions on the boundary. To be specific, from the theory of scalar valued functions we need the compact embedding of $W^{1, q}(\Omega)$ into $L^{q}(\Omega)$, i.e. Rellich's selection theorem, Korn's second inequality in $L^{q}(\Omega)$ and the so-called Lions-Lemma (3.7), which are guaranteed, if the boundary $\partial \Omega$ is locally the graph of a Lipschitz-continuous function, see e.g. $[1,20]$. Moreover, from the theory of vector fields, we need the so-called Maxwell compactness property for mixed boundary conditions, i.e., the compact embedding of $H\left(\operatorname{curl} ; \Gamma_{\tau} ; \Omega\right) \cap H\left(\operatorname{div} ; \Gamma_{\nu} ; \Omega\right)$ into $L^{2}(\Omega)$. This implies also for tensor fields the Maxwell estimate (4.2) and the Helmholtz decomposition (4.1), which are also essential tools in our arguments. These hold for Lipschitz boundaries $\partial \Omega$ as well, provided that the interface $\bar{\Gamma}_{\tau} \cap \bar{\Gamma}_{\nu}$ is Lipschitz itself. Therefore, throughout this paper we will assume generally the latter regularity.

\section{The Dev-Div-INeQuality}

In this section we shall prove the following theorem.

Theorem 3.1. Let $\Gamma_{\nu} \neq \emptyset$ and $1<q<\infty$. Then there exists a constant $C_{D D}$, such that the following estimates hold:

(i) For all $T \in L^{q}(\Omega)$

$$
\|T\|_{L^{q}(\Omega)} \leq C_{D D}\left(\|\operatorname{dev} T\|_{L^{q}(\Omega)}+\|\widetilde{\operatorname{Div}} T\|_{W^{-1, q}\left(\operatorname{Grad} ; \Gamma_{\nu} ; \Omega\right)}\right) .
$$

(ii) For all $T \in W^{q}\left(\operatorname{Div} ; \Gamma_{\nu} ; \Omega\right)$

$$
\begin{aligned}
\|T\|_{L^{q}(\Omega)} & \leq C_{D D}\left(\|\operatorname{dev} T\|_{L^{q}(\Omega)}+\|\operatorname{Div} T\|_{W^{-1, q}\left(\operatorname{Grad} ; \Gamma_{\nu} ; \Omega\right)}\right) \\
& \leq C_{D D}\left(\|\operatorname{dev} T\|_{L^{q}(\Omega)}+\|\operatorname{Div} T\|_{L^{q}(\Omega)}\right)
\end{aligned}
$$

(ii') and $\|T\|_{W^{q}(\operatorname{Div} ; \Omega)} \leq C_{D D}\left(\|\operatorname{dev} T\|_{L^{q}(\Omega)}+\|\operatorname{Div} T\|_{L^{q}(\Omega)}\right)$.

(iii) If $n=3$, for all $T \in W^{q}\left(\operatorname{Curl} ; \Gamma_{\nu} ; \Omega\right)$ it holds $\operatorname{Curl} T \in W^{q}\left(\operatorname{Div} ; \Gamma_{\nu} ; \Omega\right)$ with $\operatorname{Div} \operatorname{Curl} T=0$ and

$$
\|\operatorname{Curl} T\|_{L^{q}(\Omega)} \leq C_{D D}\|\operatorname{dev} \operatorname{Curl} T\|_{L^{q}(\Omega)} .
$$

The left hand side in (i) resp. (ii) can be replaced by

$$
\|T\|_{L^{q}(\Omega)}+\|\widetilde{\operatorname{Div}} T\|_{W^{-1, q}\left(\operatorname{Grad} ; \Gamma_{\nu} ; \Omega\right)} \quad \text { resp. } \quad\|T\|_{L^{q}(\Omega)}+\|\operatorname{Div} T\|_{W^{-1, q}\left(\operatorname{Grad} ; \Gamma_{\nu} ; \Omega\right)} .
$$

Here, the bounded linear functionals $\operatorname{Div} T$ and $\widetilde{\operatorname{Div}} T$ from $W^{-1, q}\left(\operatorname{Grad} ; \Gamma_{\nu} ; \Omega\right)$ are defined by

$$
\begin{array}{lll}
\operatorname{Div} T(v):=\langle\operatorname{Div} T, v\rangle:=\int_{\Omega} \operatorname{Div} T \cdot v \mathrm{~d} \lambda, & T \in W^{q}(\operatorname{Div} ; \Omega), \\
\widetilde{\operatorname{Div}} T(v):=\langle\widetilde{\operatorname{Div}} T, v\rangle:=-\int_{\Omega} T \cdot \operatorname{Grad} v \mathrm{~d} \lambda, & T \in L^{q}(\Omega)
\end{array}
$$

for $v \in W^{p}\left(\operatorname{Grad} ; \Gamma_{\tau} ; \Omega\right)$. Note that for $T \in W^{q}\left(\operatorname{Div} ; \Gamma_{\nu} ; \Omega\right)$ the functionals $\widetilde{\operatorname{Div}} T$ and $\operatorname{Div} T$ coincide by partial integration and

$\|\operatorname{Div} T\|_{W^{-1, q}\left(\operatorname{Grad} ; \Gamma_{\nu} ; \Omega\right)} \leq \min \left\{\|T\|_{L^{q}(\Omega)},\|\operatorname{Div} T\|_{L^{q}(\Omega)}\right\}$. 
Proof. We will follow in close lines the idea of ([2], Lem. 3.1). The key point is our subsequent Lemma 3.2, which guarantees the existence of some suitable divergence vector potential.

Let $T \in L^{q}(\Omega)$. Since by definition $T=\operatorname{dev} T+\frac{1}{n} \operatorname{tr}(T) \cdot \mathrm{Id}$, it is sufficient to estimate $\|\operatorname{tr} T\|_{L^{q}(\Omega)}$. Employing a Corollary of the Hahn-Banach's Theorem, see e.g. ([34], IV.6, Cor. 2), for every $T \in L^{q}(\Omega)$ there exists a $g \in L^{p}(\Omega)$ with $\|g\|_{L^{p}(\Omega)}=1$ and

$$
\|\operatorname{tr} T\|_{L^{q}(\Omega)}=\int_{\Omega} \operatorname{tr}(T) g \mathrm{~d} \lambda .
$$

Due to Lemma 3.2, there exists some vector field $v \in W^{p}\left(\operatorname{Grad} ; \Gamma_{\tau} ; \Omega\right)$, such that $\operatorname{div} v=g$ and the estimate $\|v\|_{W^{p}(\operatorname{Grad} ; \Omega)} \leq C\|g\|_{L^{p}(\Omega)} \leq C$ holds, where $C>0$ does not depend on $g, v$ or $T$. Thus,

$$
\begin{aligned}
\frac{1}{n}\|\operatorname{tr} T\|_{L^{q}(\Omega)} & =\frac{1}{n} \int_{\Omega} \operatorname{tr}(T) \operatorname{div} v \mathrm{~d} \lambda=\frac{1}{n} \int_{\Omega} \operatorname{tr}(T) \operatorname{Id} \cdot \operatorname{Grad} v \mathrm{~d} \lambda \\
& =\int_{\Omega} T \cdot \operatorname{Grad} v \mathrm{~d} \lambda-\int_{\Omega} \operatorname{dev} T \cdot \operatorname{Grad} v \mathrm{~d} \lambda,
\end{aligned}
$$

which shows

$$
\frac{1}{n}\|\operatorname{tr} T\|_{L^{q}(\Omega)} \leq\left(\|\operatorname{dev} T\|_{L^{q}(\Omega)}+\|\widetilde{\operatorname{Div}} T\|_{W^{-1, q}\left(\operatorname{Grad} ; \Gamma_{\nu} ; \Omega\right)}\right) \cdot \underbrace{\|v\|_{W^{p}\left(\operatorname{Grad} ; \Gamma_{\tau} ; \Omega\right)}}_{\leq C} .
$$

Hence, (i) is proved. To show (ii), let $T \in W^{q}\left(\operatorname{Div} ; \Gamma_{\nu} ; \Omega\right)$. Then, $\widetilde{\operatorname{Div}} T=\operatorname{Div} T$ as functionals from $W^{-1, q}\left(\operatorname{Grad} ; \Gamma_{\nu} ; \Omega\right)$ and $\left.\|\operatorname{Div} T\|_{W^{-1, q}\left(\operatorname{Grad} ; \Gamma_{\nu} ; \Omega\right)} \leq\|\operatorname{Div} T\|_{L^{q}(\Omega)}\right\}$. (ii') is trivial. Let $T \in W^{q}\left(\operatorname{Curl} ; \Gamma_{\nu} ; \Omega\right)$. For $n=3, \operatorname{Curl} T$ is again a quadratic tensor and the homogeneous tangential trace is mapped by the Curl operator to the homogeneous normal trace ${ }^{3}$. Thus $\operatorname{Curl} T$ is soleniodal and belongs to $W^{q}\left(\operatorname{Div} ; \Gamma_{\nu} ; \Omega\right)$. Now (iii) follows immediately by (ii) applied to $\operatorname{Curl} T$.

Lemma 3.2. Let $\Gamma_{\nu} \neq \emptyset$ and $1<p<\infty$. Then, there exists a constant $C>0$, such that for all real-valued functions $g \in L^{p}(\Omega)$ there is a vector field $v \in W^{p}\left(\operatorname{Grad} ; \Gamma_{\tau} ; \Omega\right)$ with

$$
\operatorname{div} v=g \quad \text { and } \quad\|v\|_{W^{p}(\operatorname{Grad} ; \Omega)} \leq C\|g\|_{L^{p}(\Omega)} .
$$

In the case $\Gamma_{\nu}=\emptyset$, this Lemma has been proved in ([33], Lem. 2.1.1) under the additional normalization assumption $\int_{\Omega} g \mathrm{~d} \lambda=0$. With minor modifications the same proof also works in the situation under consideration. For the convenience of the reader we shall give it in some detail.

Proof. The linear operator

$$
\underline{\operatorname{div}}: W^{p}\left(\operatorname{Grad} ; \Gamma_{\tau} ; \Omega\right) \longrightarrow L^{p}(\Omega), v \longmapsto \operatorname{div} v
$$

\footnotetext{
${ }^{3}$ For the convenience of the reader we give an illustration of this well known fact assuming a completely smooth setting: Let $E$ be a row-vector of $T, \nu$ the outward unit normal of $\Omega, \times$ the vector product in $\mathbb{R}^{3}$ and $u$ an arbitrary function with supp $u \cap \partial \Omega \subset \Gamma_{\nu}$. Using Gauss Theorem twice we compute

$$
\begin{aligned}
\int_{\partial \Omega}(\nu \cdot \operatorname{curl} E) u \mathrm{~d} o & =\int_{\Omega} \operatorname{div}(u \operatorname{curl} E) \mathrm{d} \lambda=\int_{\Omega} \operatorname{grad} u \cdot \operatorname{curl} E \mathrm{~d} \lambda=\int_{\Omega} \operatorname{grad} u \cdot \operatorname{curl} E-(\operatorname{curl} \operatorname{grad} u) \cdot E \mathrm{~d} \lambda \\
& =\int_{\Omega} \operatorname{div}(E \times \operatorname{grad} u) \mathrm{d} \lambda=\int_{\partial \Omega} \nu \cdot(E \times \operatorname{grad} u) \mathrm{d} o=\int_{\partial \Omega}(\nu \times E) \cdot \operatorname{grad} u \operatorname{do}=0
\end{aligned}
$$

if $\nu \times E=0$ on $\Gamma_{\nu}$. Since $u$ is arbitrary the normal trace of $\operatorname{curl} E$ is vanishing on $\Gamma_{\nu}$. (Using Stokes's theorem the same is proved in one line.) As often, the proof in the weak sense is much simpler: take $E \in W^{q}$ (curl; $\left.\Gamma_{\nu} ; \Omega\right)$. Then, there exists a sequence $\left(E_{n}\right) \subset C^{\infty}\left(\Gamma_{\nu}, \bar{\Omega}\right)$ with $E_{n} \rightarrow E$ in $W^{q}(\operatorname{curl} ; \Omega)$. Hence, we have $H_{n}:=\operatorname{curl} E_{n} \in C^{\infty}\left(\Gamma_{\nu}, \bar{\Omega}\right)$ with div $H_{n}=0$. Thus, $H_{n} \rightarrow H:=\operatorname{curl} E$ in $W^{q}(\operatorname{div} ; \Omega)$, which means $H \in W^{q}\left(\operatorname{div} ; \Gamma_{\nu} ; \Omega\right)$ with $\operatorname{div} H=0$.
} 
is bounded, i.e.

$$
\|\underline{\operatorname{div}} v\|_{L^{p}(\Omega)} \leq C_{1}\|v\|_{W^{p}(\operatorname{Grad} ; \Omega)}
$$

holds for all $v \in W^{P}\left(\operatorname{Grad} ; \Gamma_{\tau} ; \Omega\right)$. We identify $\left(L^{p}(\Omega)\right)^{\prime}=L^{q}(\Omega)$. Further, we consider the dual operator of div,

$$
\underline{\operatorname{div}}^{\prime}=-\operatorname{grad}: L^{q}(\Omega) \longrightarrow\left(W^{p}\left(\operatorname{Grad} ; \Gamma_{\tau} ; \Omega\right)\right)^{\prime}=W^{-1, q}\left(\operatorname{Grad} ; \Gamma_{\nu} ; \Omega\right),
$$

defined by

$$
-\langle\underline{\operatorname{grad}} u, v\rangle:=\int_{\Omega} u \operatorname{div} v \mathrm{~d} \lambda
$$

for all $v \in W^{p}\left(\operatorname{Grad} ; \Gamma_{\tau} ; \Omega\right)$ and all $u \in L^{q}(\Omega)$. Here again, the brackets $\langle\cdot, \cdot\rangle$ denote the duality pairing of $W^{p}\left(\operatorname{Grad} ; \Gamma_{\tau} ; \Omega\right)$ and $W^{-1, q}\left(\mathrm{Grad} ; \Gamma_{\nu} ; \Omega\right)$. Utilizing (3.3) and the definition of the norm in the dual space we obtain the continuity of grad, i.e.,

$$
\|\underline{\operatorname{grad}} u\|_{W^{-1, q}\left(\operatorname{Grad} ; \Gamma_{\nu} ; \Omega\right)} \leq C_{1}\|u\|_{L^{q}(\Omega)} .
$$

We will show that also the reversed inequality holds true: there exists a constant $C_{2}>0$, such that for all $u \in L^{q}(\Omega)$

$$
\|u\|_{L^{q}(\Omega)} \leq C_{2}\|\underline{\operatorname{grad}} u\|_{W^{-1, q}\left(\operatorname{Grad} ; \Gamma_{\nu} ; \Omega\right)} .
$$

To prove (3.4) we use the usual contradiction argument: assume the inequality is false, then there exists a sequence $\left(u_{j}\right) \subset L^{q}(\Omega)$ with

$$
\left\|u_{j}\right\|_{L^{q}(\Omega)}=1 \text { for all } j \quad \text { and } \quad \lim _{j \rightarrow \infty}\|\underline{\operatorname{grad}} u\|_{W^{-1, q}\left(\operatorname{Grad} ; \Gamma_{\nu} ; \Omega\right)}=0 .
$$

Since $\left(u_{j}\right)$ is bounded in $L^{q}(\Omega)$, by weak compactness there exists a subsequence of $\left(u_{j}\right)$, also called $\left(u_{j}\right)$, and a $u \in L^{q}(\Omega)$, such that

$$
u_{j} \rightarrow u \quad \text { weakly in } L^{q}(\Omega) .
$$

Since for all $v \in W^{p}\left(\mathrm{Grad} ; \Gamma_{\tau} ; \Omega\right)$

$$
\begin{aligned}
|\langle\underline{\operatorname{grad}} u, v\rangle| & =\left|\int_{\Omega} u \operatorname{div} v \mathrm{~d} \lambda\right|=\lim _{j \rightarrow \infty}\left|\int_{\Omega} u_{j} \operatorname{div} v \mathrm{~d} \lambda\right| \\
& =\lim _{j \rightarrow \infty}\left|\left\langle\operatorname{grad} \underline{\operatorname{rad}} u_{j}, v\right\rangle\right| \leq \lim _{j \rightarrow \infty}\left\|\underline{\operatorname{grad}} u_{j}\right\|_{W^{-1, q}\left(\operatorname{Grad} ; \Gamma_{\nu} ; \Omega\right)}\|v\|_{\left.W^{p}\left(\operatorname{Grad} ; \Gamma_{\tau} ; \Omega\right)\right)}=0,
\end{aligned}
$$

we conclude $\operatorname{grad} u=0$, which implies $\operatorname{grad} u=0$ in the distributional sense and hence by the fundamental lemma $u=$ const, see also (e.g. [33], II, (1.7.18)). As $\Gamma_{\nu} \neq \emptyset$ is relatively open, there exists a vector field $\hat{v} \in W^{p}\left(\operatorname{Grad} ; \Gamma_{\tau} ; \Omega\right)$ such that

$$
\int_{\Omega} \operatorname{div} \hat{v} \mathrm{~d} \lambda \neq 0
$$

Employing this, (3.6) and $u=$ const we conclude $u=0$ since

$$
0=\langle\operatorname{grad} u, \hat{v}\rangle=u \int_{\Omega} \operatorname{div} \hat{v} \mathrm{~d} \lambda .
$$

Remarkably, the operator grad, although being a kind of differential operator, does not vanish on constant functions.

Following ([1], Thm. 6.3) the embedding $W^{p}(\operatorname{grad} ; \Omega) \hookrightarrow L^{q}(\Omega)$ is compact. Hence, of course also $W^{p}\left(\operatorname{grad} ; \Gamma_{\tau} ; \Omega\right) \hookrightarrow L^{q}(\Omega)$ is compact. Using ([34], X. 4), the dual embedding

$$
L^{q}(\Omega) \hookrightarrow W^{-1, q}\left(\operatorname{grad} ; \Gamma_{\nu} ; \Omega\right)=\left(W^{p}\left(\operatorname{grad} ; \Gamma_{\tau} ; \Omega\right)\right)^{\prime},
$$


defined by $\langle f, w\rangle=\int_{\Omega} f \cdot w \mathrm{~d} \lambda$ for all $f \in L^{q}(\Omega)$ and $w \in W^{p}\left(\operatorname{grad} ; \Gamma_{\tau} ; \Omega\right)$, is compact as well. Thus, we can select a subsequence, again denoted by $\left(u_{j}\right)$, which converges to some $\hat{u} \in W^{-1, q}\left(\operatorname{grad} ; \Gamma_{\nu} ; \Omega\right)$ in $W^{-1, q}\left(\operatorname{grad} ; \Gamma_{\nu} ; \Omega\right)$. As we have seen, $\left(u_{j}\right)$ also converges weakly in $L^{q}(\Omega)$ to $u=0$ and therefore we get $\hat{u}=0$. Now we use the so-called Lions-Lemma from [20] (concerning the history of the Lions-Lemma, see also [10]): There is a positive constant $C_{3}$, such that for all $u \in L^{q}(\Omega)$

$$
\|u\|_{L^{q}(\Omega)} \leq C_{3}\left(\|\operatorname{grad} u\|_{W^{-1, q}(\operatorname{Grad} ; \Omega)}+\|u\|_{W^{-1, q}(\operatorname{grad} ; \Omega)}\right) .
$$

The norms of dual spaces $W^{-1, q}\left(\operatorname{grad} ; \Gamma_{\nu} ; \Omega\right)$ and $W^{-1, q}\left(\operatorname{Grad} ; \Gamma_{\nu} ; \Omega\right)$ are stronger than the norms of $W^{-1, q}(\operatorname{grad} ; \Omega)$ and $W^{-1, q}(\operatorname{Grad} ; \Omega)$. Hence, we can estimate

$$
\begin{aligned}
1=\left\|u_{j}\right\|_{L^{q}(\Omega)} & \leq C_{3}\left(\left\|\operatorname{grad} u_{j}\right\|_{W^{-1, q}(\operatorname{Grad} ; \Omega)}+\left\|u_{j}\right\|_{W^{-1, q}(\operatorname{grad} ; \Omega)}\right) \\
& \leq C_{3}\left(\left\|\underline{\operatorname{grad}} u_{j}\right\|_{W^{-1, q}\left(\operatorname{Grad} ; \Gamma_{\nu} ; \Omega\right)}+\left\|u_{j}\right\|_{W^{-1, q}\left(\operatorname{grad} ; \Gamma_{\nu} ; \Omega\right)}\right) \longrightarrow 0
\end{aligned}
$$

for $j \rightarrow \infty$, in contradiction to (3.5). Thus (3.4) is proved.

By (3.4), the range $R(\mathrm{grad})$ of the operator grad is a closed subspace of $W^{-1, q}\left(\mathrm{Grad} ; \Gamma_{\nu} ; \Omega\right)$. Since $R(\underline{\operatorname{grad}})$ is the range of the dual operator of div, the closed range theorem (see e.g. [34], VII.5), yields that the range $R(\underline{\text { div }})$ is also closed and we have

$$
R(\underline{\operatorname{div}})=\left\{f \in L^{p}(\Omega): \int_{\Omega} f \cdot u \mathrm{~d} \lambda=0 \quad \text { for all } \quad u \in N(\underline{\operatorname{grad}})\right\},
$$

where $N(\underline{\operatorname{grad}})$ denotes the kernel of the operator grad. We have already shown above that grad $u=0$ implies $u=0$, i.e. $N(\underline{\mathrm{grad}})=\{0\}$. Therefore,

$$
R(\underline{\operatorname{div}})=L^{p}(\Omega) .
$$

In order to get the estimate in (3.2), we consider the quotient space

$$
W^{p}\left(\operatorname{Grad} ; \Gamma_{\tau} ; \Omega\right) / N(\underline{\mathrm{div}}):=\left\{[v] \mid v \in W^{p}\left(\mathrm{Grad} ; \Gamma_{\tau} ; \Omega\right)\right\},
$$

with $[v]:=v+N(\underline{\operatorname{div}}), v \in W^{p}\left(\operatorname{Grad} ; \Gamma_{\tau} ; \Omega\right)$ and the associated norm

$$
\|[v]\|_{W^{p}\left(\operatorname{Grad} ; \Gamma_{\tau} ; \Omega\right) / N(\underline{\mathrm{div}})}:=\inf _{w \in N(\underline{\mathrm{div}})}\|v+w\|_{W^{p}(\operatorname{Grad} ; \Omega)} .
$$

Thus, the linear operator

$$
\overline{\operatorname{div}}: W^{p}\left(\operatorname{Grad} ; \Gamma_{\tau} ; \Omega\right) / N(\underline{\operatorname{div}}) \longrightarrow L^{p}(\Omega), \quad[v] \longmapsto \operatorname{div} v
$$

is well-defined, bijective and bounded. According to the bounded inverse theorem (see e.g. [34], II. 5), the inverse operator $\overline{\operatorname{div}}^{-1}$, mapping $L^{p}(\Omega)$ to $W^{p}\left(\mathrm{Grad} ; \Gamma_{\tau} ; \Omega\right) / N(\underline{\operatorname{div}})$ is bounded. Hence there exists a constant $C_{4}>0$, such that for all $g \in L^{p}(\Omega)$ with $g=\operatorname{div} v$ and $v \in W^{p}\left(\underline{\operatorname{Grad}} ; \Gamma_{\tau} ; \Omega\right)$

$$
\inf _{w \in N(\underline{\text { div }})}\|v+w\|_{W^{p}(\operatorname{Grad} ; \Omega)} \leq C_{4}\|g\|_{L^{p}(\Omega)} .
$$

Choosing now any constant $C_{5}>C_{4}$, then for all $g \in L^{p}(\Omega)$ there exists $v \in W^{p}\left(\operatorname{Grad} ; \Gamma_{\tau} ; \Omega\right)$ with $\operatorname{div} v=g$ and

$$
\|v\|_{W^{p}(\operatorname{Grad} ; \Omega)} \leq C_{5}\|g\|_{L^{p}(\Omega)}
$$

which completes the proof. 
Finally, we formulate (3.4) separately. For this, we recall $\nabla:=\operatorname{grad}$, i.e.,

$$
\nabla: L^{q}(\Omega) \rightarrow W^{-1, q}\left(\operatorname{Grad} ; \Gamma_{\nu} ; \Omega\right)
$$

defined by

$$
\langle\nabla u, v\rangle:=-\int_{\Omega} u \operatorname{div} v \mathrm{~d} \lambda
$$

for all $u \in L^{q}(\Omega)$ and all $v \in W^{p}\left(\operatorname{Grad} ; \Gamma_{\tau} ; \Omega\right)$.

Lemma 3.3. There exists a constant $c>0$, such that for all $u \in L^{q}(\Omega)$

$$
c^{-1}\|u\|_{L^{q}(\Omega)} \leq\|\nabla u\|_{W^{-1, q}\left(\operatorname{Grad} ; \Gamma_{\nu} ; \Omega\right)} \leq c\|u\|_{L^{q}(\Omega)} .
$$

\section{The DevSym-Curl-Inequality}

Sym-Curl-estimates have been established recently in a series of papers by some of the present authors and have been shown to hold true also for mixed boundary conditions, see [29] for $n=3$ and [26] for arbitrary dimensions. For these results it is crucial that the domain $\Omega$ allows for the so-called Maxwell compactness property, i.e. the compact embedding of $H\left(\operatorname{curl} ; \Gamma_{\tau} ; \Omega\right) \cap H\left(\operatorname{div} ; \Gamma_{\nu} ; \Omega\right)$ into $L^{2}(\Omega)$, and the so-called Maxwell approximation property, see [26]. These two properties ensure that the Helmholtz decomposition (also for tensor fields) holds true, see [26, 29]:

$$
L^{2}(\Omega)=\operatorname{Grad} H\left(\operatorname{Grad} ; \Gamma_{\tau} ; \Omega\right) \oplus \mathcal{H}(\Omega) \oplus \operatorname{Curl} H\left(\operatorname{Curl} ; \Gamma_{\nu} ; \Omega\right),
$$

where $\mathcal{H}(\Omega)$ is the space of harmonic Dirichlet-Neumann-tensors, i.e., the space of tensors $T$ belonging to $H\left(\operatorname{Curl} ; \Gamma_{\tau} ; \Omega\right) \cap H\left(\operatorname{Div} ; \Gamma_{\nu} ; \Omega\right)$ with $\operatorname{Curl} T=0$ and $\operatorname{Div} T=0$, and $\oplus$ denotes orthogonality in $L^{2}(\Omega)$. Due to the Maxwell compactness property, the unit ball in $\mathcal{H}(\Omega)$ is compact and hence the space $\mathcal{H}(\Omega)$ has finite dimension, the dimension depending on topological properties of the domain. In consequence of the Maxwell compactness property, a Poincaré-type Maxwell estimate is achieved by a standard indirect argument, i.e.

$$
\|T\|_{L^{2}(\Omega)} \leq C_{m}\left(\|\operatorname{Curl} T\|_{L^{2}(\Omega)}+\|\operatorname{Div} T\|_{L^{2}(\Omega)}\right)
$$

for all $T \in H\left(\operatorname{Curl} ; \Gamma_{\tau} ; \Omega\right) \cap H\left(\operatorname{Div} ; \Gamma_{\nu} ; \Omega\right)$ perpendicular to $\mathcal{H}(\Omega)$, see [29]. Both, the Maxwell compactness property and the Maxwell approximation property have been proved to be satisfied, if the underlying domain $\Omega$ has a Lipschitz boundary, and in addition the interface between the two kinds of boundaries

$$
\bar{\Gamma}_{\tau} \cap \bar{\Gamma}_{\nu} \quad \text { is also Lipschitz, }
$$

see $[16,17]$ and the discussion in $[26,29]$.

In order to deal with the influence of possible harmonic Dirichlet-Neumann-tensors, in ([29], Def. 10) a further technical condition on the domain $\Omega$ and the topology of $\Omega$ is imposed:

Definition 4.1. $\Omega$ is called sliceable, if there exist a natural number $J \in \mathbb{N}$ and $\Omega_{j} \subset \Omega, j=1, \ldots, J$, such that $\Omega \backslash\left(\Omega_{1} \cup \cdots \cup \Omega_{J}\right)$ is a null set and for $j=1, \ldots, J$

(i) $\Omega_{j}$ are open, disjoint and simply connected Lipschitz subdomains of $\Omega$,

(ii) $\Gamma_{\tau, j}:=\operatorname{int}_{\text {rel }}\left(\bar{\Omega}_{j} \cap \Gamma_{\tau}\right) \neq \emptyset$, if $\Gamma_{\tau} \neq \emptyset$.

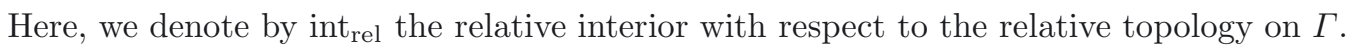

First we prove:

Lemma 4.2. Let $n \geq 3$ and $\Gamma_{\tau} \neq \emptyset$ or $n=2$ and $\Gamma_{\tau}=\partial \Omega$. Then, there is a constant $C_{D S G}$, such that for all $v \in H\left(\operatorname{Grad} ; \Gamma_{\tau} ; \Omega\right)$

$$
\|\operatorname{Grad} v\|_{L^{2}(\Omega)} \leq C_{D S G}\|\operatorname{dev} \operatorname{sym} \operatorname{Grad} v\|_{L^{2}(\Omega)} .
$$



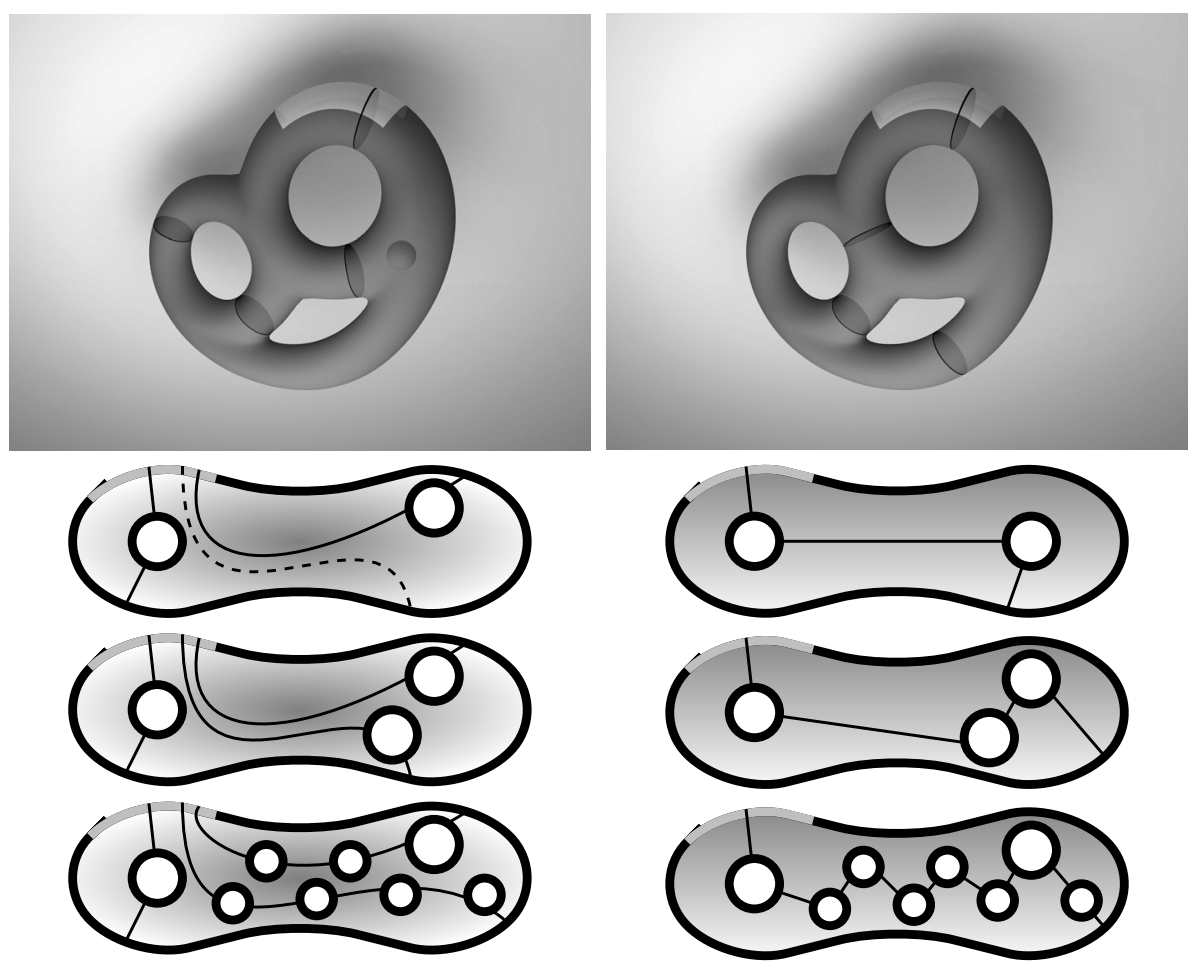

Figure 1 . Some ways to 'cut' sliceable domains $\Omega$ in $\mathbb{R}^{3}$ and $\mathbb{R}^{2}$ into two $(J=2)$ or more $(J=3,4)$ 'pieces'. The boundary part $\Gamma_{\tau}$ is colored in light gray. Roughly speaking, a domain is sliceable if it can be cut into finitely many simply connected Lipschitz pieces $\Omega_{j}$, i.e., any closed curve inside some piece $\Omega_{j}$ is homotop to a point, this is, one has to cut all 'handles'. In three and higher dimensions, holes inside $\Omega$ are permitted, but this is forbidden in the twodimensional case. Note that, in these examples it is always possible to slice $\Omega$ into two $(J=2)$ pieces.

The proof of Lemma 4.2 relies only on the estimate (1.9), i.e., an improved version of Korn's second inequality, Rellich's selection theorem and the control of the kernel of dev sym Grad through the boundary condition. On this account, a representation formula for elements in this kernel is needed, which is given in the Appendix of this paper. The case $n=2$ with full boundary condition is already proved in the Appendix of [29] and a counterexample to (4.4) for the case $n=2$ without the full boundary condition will be given in Section 6 .

Proof. In a first step, we prove

$$
\left(v \in H\left(\operatorname{Grad} ; \Gamma_{\tau} ; \Omega\right) \wedge \quad \operatorname{dev} \operatorname{sym} \operatorname{Grad} v=0\right) \quad \Rightarrow \quad v=0 .
$$

We utilize the following representation of the kernel which is proved in the Appendix: There are vectors $\bar{v}, \bar{w} \in$ $\mathbb{R}^{n}$, a real number $\bar{u} \in \mathbb{R}$ and a skew-symmetric matrix $\bar{A} \in \mathfrak{s o}(n)$, such that

$$
\begin{aligned}
v(x) & =u(x) x-\frac{1}{2}|x|^{2} \bar{w}+\bar{A} x+\bar{v}, \\
\operatorname{Grad} v(x) & =u(x) \operatorname{Id}+A(x),
\end{aligned}
$$


holds for all $x \in \bar{\Omega}$, where

$$
u(x)=\bar{w} \cdot x+\bar{u}, \quad A_{i j}(x)=\sum_{k=1}^{n} \bar{a}_{i j k} x_{k}+\bar{A}_{i j}
$$

and

$$
\bar{a}_{i j k}=\left\{\begin{array}{lll}
0 & \text { if } \quad i \neq j, i \neq k, k \neq j \\
0 & \text { if } \quad i=j, \\
\bar{w}_{j} & \text { if } \quad k=i, i \neq j, \\
-\bar{w}_{i} & \text { if } \quad k=j, k \neq i .
\end{array}\right.
$$

In particular, $A(x)$ is skew-symmetric and the dimension of the kernel of dev sym Grad is $(n+1)(n+2) / 2$. Due to this formula, $v$ is a smooth vector field on $\bar{\Omega}$. Let $x \in \Gamma_{\tau}$ and $\tau \in \mathbb{R}^{n}, \tau \neq 0$, tangential to $\Gamma_{\tau}$ in $x$. Since $v \in H\left(\operatorname{Grad} ; \Gamma_{\tau} ; \Omega\right)$, we have $\operatorname{Grad} v \in H\left(\operatorname{Curl} ; \Gamma_{\tau} ; \Omega\right)$, i.e.

$$
\operatorname{Grad} v(x) \tau=0 .
$$

Therefore, if $x \in \Gamma_{\tau}$, then $\operatorname{Grad} v(x)$ does not have full rank. By (4.7) and since $\tau \cdot A(x) \tau=0$

$$
0=|\operatorname{Grad} v(x) \tau|^{2}=u^{2}(x)|\tau|^{2}+|A(x) \tau|^{2}
$$

holds with $u$ and $A$ from (4.8). Hence $u(x)=0$. Therefore, (4.8) implies necessarily

$$
0=u(x)=\bar{w} \cdot x+\bar{u} \text { for all } x \in \Gamma_{\tau} .
$$

On the other hand, if $u(x)=0$, then $\operatorname{Grad} v(x)$ has not full rank, since $A(x)$ is skew-symmetric. Thus, for all $x \in \Gamma_{\tau}$ the matrix $\operatorname{Grad} v(x)$ does not have full rank, if and only if (4.11) holds. If $\bar{w} \neq 0$, then by (4.11) $\Gamma_{\tau} \subset E$, where $E$ denotes the affine hypersurface defined by equation (4.11). On the other hand, for all $x \in \Gamma_{\tau} \subset E$, due to the representation formula (4.6) and (4.11), we get

$$
v(x)=-\frac{1}{2}|x|^{2} \bar{w}+\bar{A} x+\bar{v}=0,
$$

describing for $\bar{w} \neq 0$ a quadratic surface and not a hypersurface. This proves $\bar{w}=0$ and hence $u=\bar{u}=0$. Consequently, on $\Gamma_{\tau}$

$$
v(x)=\bar{A} x+\bar{v}=0,
$$

yielding $\bar{A}=0$ and $\bar{v}=0$, since otherwise the solution set of (4.13) is an affine surface with co-dimension codim $\geq 2$, recall that $\bar{A}$ is skew-symmetric. But such a surface cannot contain an open and non-empty subset of a Lipschitz-continuous boundary. Therefore (4.5) is proved.

In the second step we utilize 1.9 from ([11], Thm. 1.1) and carry out the usual conclusion by contradiction. Assume the estimate (4.4) is false, then there exists a sequence $\left(v_{j}\right) \subset H\left(\mathrm{Grad} ; \Gamma_{\tau} ; \Omega\right)$ with $\left\|\operatorname{Grad} v_{j}\right\|_{L^{2}(\Omega)}=1$ and

$$
\left\|\operatorname{dev} \operatorname{sym} \operatorname{Grad} v_{j}\right\|_{L^{2}(\Omega)}<\frac{1}{j}
$$

for all $j \in \mathbb{N}$. According to (1.9) the sequence of norms $\left\|v_{j}\right\|_{L^{2}(\Omega)}$ is bounded from below, i.e. there exists $J \in \mathbb{N}$ and a constant $C>0$, such that

$$
\left\|v_{j}\right\|_{L^{2}(\Omega)} \geq C \text { for all } j \geq J .
$$

Utilizing Poincaré's inequality and $\left\|\operatorname{Grad} v_{j}\right\|_{L^{2}(\Omega)}=1$, the sequence $\left(v_{j}\right)$ is bounded in $H(\mathrm{Grad} ; \Omega)$. Employing Rellich's selection theorem there is a subsequence of $\left(v_{j}\right)$, again called $\left(v_{j}\right)$, and $v \in H\left(\mathrm{Grad} ; \Gamma_{\tau} ; \Omega\right)$ such that

$$
\begin{aligned}
v_{j} & \rightarrow v \quad \text { strongly in } L^{2}(\Omega), \\
\operatorname{Grad} v_{j} & \rightarrow \operatorname{Grad} v \quad \text { weakly in } L^{2}(\Omega) .
\end{aligned}
$$


Hence dev $\operatorname{sym} \operatorname{Grad} v_{j}$ converges weakly to $\operatorname{dev} \operatorname{sym} \operatorname{Grad} v$ and due to weak lower semi-continuity of the norm and (4.14) we conclude

$$
\|\operatorname{dev} \operatorname{sym} \operatorname{Grad} v\|_{L^{2}(\Omega)} \leq \liminf _{j \rightarrow \infty}\left\|\operatorname{dev} \operatorname{sym} \operatorname{Grad} v_{j}\right\|_{L^{2}(\Omega)}=0 .
$$

According to (4.5), this implies $v=0$, in contradiction to (4.15) und (4.16). Therefore, Lemma 4.2 is proved.

Now, we can prove the DevSym-Curl-inequality:

Theorem 4.3. Let $n \geq 3, \Omega \subset \mathbb{R}^{n}$ be a slicable domain and $\Gamma_{\tau} \neq \emptyset$. Then, there is a positive constant $C_{D S C}$, such that for all $T \in H\left(\mathrm{Curl} ; \Gamma_{\tau} ; \Omega\right)$

$$
\|T\|_{L^{2}(\Omega)} \leq C_{D S C}\left(\|\operatorname{dev} \operatorname{sym} T\|_{L^{2}(\Omega)}+\|\operatorname{Curl} T\|_{L^{2}(\Omega)}\right) .
$$

We note that Theorem 4.3 remains true if $n=2$ and $\Gamma_{\tau}=\partial \Omega$ since Lemma 4.2 holds in this case as well. Moreover, with (4.17) also

$$
\|T\|_{L^{2}(\Omega)}+\|\operatorname{Curl} T\|_{L^{2}(\Omega)} \leq C_{D S C}\left(\|\operatorname{dev} \operatorname{sym} T\|_{L^{2}(\Omega)}+\|\operatorname{Curl} T\|_{L^{2}(\Omega)}\right)
$$

holds.

Proof. We combine the proof of the Sym-Curl-inequality (1.2) from the papers $[26,29]$ with Lemma 4.2. Let $T \in H\left(\mathrm{Curl} ; \Gamma_{\tau} ; \Omega\right)$. Using the Helmholtz decomposition from [26] we have the orthogonal sum

$$
T=R+S \in H\left(\operatorname{Curl}_{0} ; \Gamma_{\tau} ; \Omega\right) \oplus \operatorname{Curl} H\left(\operatorname{Curl} ; \Gamma_{\nu} ; \Omega\right),
$$

where $R \in\left(\operatorname{Curl}_{0} ; \Gamma_{\tau} ; \Omega\right)$, if and only if $R \in H\left(\operatorname{Curl} ; \Gamma_{\tau} ; \Omega\right)$ and $\operatorname{Curl} R=0$. Note, that in general $R \in H\left(\operatorname{Curl}_{0} ; \Gamma_{\tau} ; \Omega\right)$ does not imply $R=\operatorname{Grad} v$ with $v \in H\left(\operatorname{Grad} ; \Gamma_{\tau} ; \Omega\right)$, since, depending on topological properties of the domain $\Omega$, some harmonic-Dirichlet-Neumann tensor fields could be involved. In order to deal with this possibility, we slice the domain $\Omega$ according to Definition 4.1 and set

$$
R=\sum_{j=1}^{J} \chi_{j} R_{j},
$$

where $R_{j}:=\left.R\right|_{\Omega_{j}}$ and $\chi_{j}$ is the indicator-function of $\Omega_{j}$. In the proofs of ([29], Lems. 9 and 12) it is shown, that there are non-empty and relatively open connected subsets $\widetilde{\Gamma}_{\tau, j} \subset \Gamma_{\tau, j}$ and vector fields $v_{j} \in H\left(\operatorname{Grad} ; \widetilde{\Gamma}_{\tau, j} ; \Omega_{j}\right)$ such that Grad $v_{j}=R_{j}$. Now we apply (4.4) to $v_{j}$ and get

$$
\begin{aligned}
\|T\|_{L^{2}(\Omega)}^{2} & =\|R\|_{L^{2}(\Omega)}^{2}+\|S\|_{L^{2}(\Omega)}^{2}=\sum_{j=1}^{J}\left\|R_{j}\right\|_{L^{2}\left(\Omega_{j}\right)}^{2}+\|S\|_{L^{2}(\Omega)}^{2} \\
& \leq C \sum_{j=1}^{J}\left\|\operatorname{dev} \operatorname{sym} R_{j}\right\|_{L^{2}\left(\Omega_{j}\right)}^{2}+\|S\|_{L^{2}(\Omega)}^{2}=C\|\operatorname{dev} \operatorname{sym} R\|_{L^{2}(\Omega)}^{2}+\|S\|_{L^{2}(\Omega)}^{2} \\
& \leq C\|\operatorname{dev} \operatorname{sym} T\|_{L^{2}(\Omega)}^{2}+C\|\operatorname{dev} \operatorname{sym} S\|_{L^{2}(\Omega)}^{2}+\|S\|_{L^{2}(\Omega)}^{2} \\
& \leq C\|\operatorname{dev} \operatorname{sym} T\|_{L^{2}(\Omega)}^{2}+C\|S\|_{L^{2}(\Omega)}^{2} .
\end{aligned}
$$

Concerning the $S$-part, we note that $\operatorname{Curl} T=\operatorname{Curl} S$ and $S \in H\left(\operatorname{Curl} ; \Gamma_{\tau} ; \Omega\right)$ since $T$ and $R$ belong to $H\left(\mathrm{Curl} ; \Gamma_{\tau} ; \Omega\right)$. Moreover, since

$$
\operatorname{Curl} H\left(\operatorname{Curl} ; \Gamma_{\nu} ; \Omega\right) \subset H\left(\operatorname{Div}_{0} ; \Gamma_{\nu} ; \Omega\right) \cap \mathcal{H}(\Omega)^{\perp}
$$


we even have $S \in H\left(\operatorname{Curl} ; \Gamma_{\tau} ; \Omega\right) \cap H\left(\operatorname{Div}_{0} ; \Gamma_{\nu} ; \Omega\right) \cap \mathcal{H}(\Omega)^{\perp}$. By means of the Maxwell's inequality (4.2) and since Div $S=0$ we estimate

$$
\|S\|_{L^{2}(\Omega)} \leq C_{m}\|\operatorname{Curl} S\|_{L^{2}(\Omega)}=C_{m}\|\operatorname{Curl} T\|_{L^{2}(\Omega)} .
$$

Combining (4.19) and (4.18) yields

$$
\|T\|_{L^{2}(\Omega)} \leq C\|\operatorname{dev} \operatorname{sym} T\|_{L^{2}(\Omega)}+C\|\operatorname{Curl} T\|_{L^{2}(\Omega)},
$$

completing the proof.

\section{The Sym-DevCurl- and DevSym-DevCurl-inequalities}

Now, we combine the Dev-Div-inequality with the Sym-Curl-inequality and the DevSym-Curl-inequality. For this, we need $n=3$ since only then $\operatorname{Curl} T$ is again quadratic.

Theorem 5.1. Let $\Omega \subset \mathbb{R}^{3}$ be a slicable domain and $\Gamma_{\tau} \neq \emptyset$. Then, there are positive constants $C_{S D C}$ and $C_{D S D C}$, such that for all $T \in H\left(\operatorname{Curl} ; \Gamma_{\tau} ; \Omega\right)$

$$
\begin{aligned}
& \|T\|_{L^{2}(\Omega)} \leq C_{S D C}\left(\|\operatorname{sym} T\|_{L^{2}(\Omega)}+\|\operatorname{dev} \operatorname{Curl} T\|_{L^{2}(\Omega)}\right), \\
& \|T\|_{L^{2}(\Omega)} \leq C_{D S D C}\left(\|\operatorname{dev} \operatorname{sym} T\|_{L^{2}(\Omega)}+\|\operatorname{dev} \operatorname{Curl} T\|_{L^{2}(\Omega)}\right)
\end{aligned}
$$

and

$$
\begin{aligned}
\|T\|_{L^{2}(\Omega)}+\|\operatorname{Curl} T\|_{L^{2}(\Omega)} & \leq C_{S D C}\left(\|\operatorname{sym} T\|_{L^{2}(\Omega)}+\|\operatorname{dev} \operatorname{Curl} T\|_{L^{2}(\Omega)}\right), \\
\|T\|_{L^{2}(\Omega)}+\|\operatorname{Curl} T\|_{L^{2}(\Omega)} & \leq C_{D S D C}\left(\|\operatorname{dev} \operatorname{sym} T\|_{L^{2}(\Omega)}+\|\operatorname{dev} \operatorname{Curl} T\|_{L^{2}(\Omega)}\right) .
\end{aligned}
$$

Proof. Combine Theorem 4.3 with Theorem 3.1 (ii).

\section{KeRnELS AND COUNTEREXAMPLES}

It is illuminating to see, how the kernels of the inequalities are controlled on, say, the space of smooth compactly supported tensor fields. Of course, some of the given arguments are well known. In the following we always assume that $T$ is such a smooth tensor field with compact support in $\Omega \subset \mathbb{R}^{3}$.

\subsection{The kernel of the Dev-Div-inequality}

Consider some $T$ in the kernel of the Dev-Div-inequality, i.e., $\operatorname{dev} T=0$ and $\operatorname{Div} T=0$. Since $\operatorname{dev} T=0$ we have $T=u \cdot$ Id. But therefore $\operatorname{Div} T=\operatorname{grad} u=0$ and we conclude $u=$ const. Since $u$ and $T$ are compactly supported, $u=0$ and $T=0$ in $\Omega$.

\subsection{The kernel of the Sym-Curl-inequality}

Consider some $T$ in the kernel of the Sym-Curl-inequality, i.e., sym $T=0$ and $\operatorname{Curl} T=0$. Since $\operatorname{sym} T=0$ we conclude $T(x)=A(x) \in \mathfrak{s o}(3)$, say

$$
\begin{gathered}
A=\left(\begin{array}{ccc}
0 & -a_{3} & a_{2} \\
a_{3} & 0 & -a_{1} \\
-a_{2} & a_{1} & 0
\end{array}\right) \text { and } \\
\operatorname{Curl} A=\left(\begin{array}{ccc}
\partial_{2} a_{2}+\partial_{3} a_{3} & -\partial_{1} a_{2} & -\partial_{1} a_{3} \\
-\partial_{2} a_{1} & \partial_{3} a_{3}+\partial_{1} a_{1} & -\partial_{2} a_{3} \\
-\partial_{3} a_{1} & -\partial_{3} a_{2} & \partial_{1} a_{1}+\partial_{2} a_{2}
\end{array}\right)
\end{gathered}
$$

with a smooth and compactly supported vector field $a=\left(a_{1}, a_{2}, a_{3}\right)^{\top}$. Hence $\operatorname{Curl} A=0$ implies $\operatorname{Grad} a=0$ and thus $a=0$ and $T=A=0$, see also [22]. 


\subsection{The kernel of the DevSym-DevCurl-inequality}

Regarding the DevSym-DevCurl-inequality the situation gets more involved. Let us assume dev sym $T=0$ and $\operatorname{dev} \operatorname{Curl} T=0$. Then

$$
\begin{aligned}
T(x) & =u(x) \cdot \operatorname{Id}+A(x), \\
\operatorname{Curl}(u(x) \cdot \operatorname{Id})+\operatorname{Curl} A(x)=\operatorname{Curl} T(x) & =y(x) \cdot \operatorname{Id}
\end{aligned}
$$

with smooth and compactly supported functions $u, y$ and with $a, A$ as above. Now

$$
\operatorname{Curl}(u \cdot \operatorname{Id})=\left(\begin{array}{ccc}
0 & \partial_{3} u & -\partial_{2} u \\
-\partial_{3} u & 0 & \partial_{1} u \\
\partial_{2} u & -\partial_{1} u & 0
\end{array}\right)
$$

is a skew-symmetric matrix. Therefore, $\operatorname{sym} \operatorname{Curl} A=y \cdot \operatorname{Id}$ and hence by (6.2)

$$
\partial_{1} a_{2}+\partial_{2} a_{1}=\partial_{2} a_{3}+\partial_{3} a_{2}=\partial_{3} a_{1}+\partial_{1} a_{3}=0
$$

and

$$
\partial_{2} a_{2}+\partial_{3} a_{3}=\partial_{3} a_{3}+\partial_{1} a_{1}=\partial_{1} a_{1}+\partial_{2} a_{2}=y .
$$

The second series of equations yields

$$
\partial_{1} a_{1}=\partial_{2} a_{2}=\partial_{3} a_{3}=\frac{y}{2} \quad \text { as well as } \quad 2 \operatorname{div} a=3 y .
$$

By means of comparison of the skew-symmetric parts of equation (6.4), utilizing (6.1) and (6.5), we conclude that

$$
\operatorname{grad} u=\left(\begin{array}{c}
\partial_{2} a_{3} \\
\partial_{3} a_{1} \\
\partial_{1} a_{2}
\end{array}\right)
$$

and thus, employing (6.6)

$$
0=\operatorname{curl} \operatorname{grad} u=\operatorname{curl}\left(\begin{array}{c}
\partial_{2} a_{3} \\
\partial_{3} a_{1} \\
\partial_{1} a_{2}
\end{array}\right)=-\left(\begin{array}{c}
\left(\partial_{2}^{2}+\partial_{3}^{2}\right) a_{1} \\
\left(\partial_{3}^{2}+\partial_{1}^{2}\right) a_{2} \\
\left(\partial_{1}^{2}+\partial_{2}^{2}\right) a_{3}
\end{array}\right)
$$

With (6.10) and (6.8) we obtain

$$
\Delta a=-\frac{1}{2} \operatorname{grad} y=-\frac{1}{3} \operatorname{grad} \operatorname{div} a
$$

Furthermore, due to $(6.6)$

$$
\operatorname{curl} a=2\left(\begin{array}{c}
\partial_{2} a_{3} \\
\partial_{3} a_{1} \\
\partial_{1} a_{2}
\end{array}\right)
$$

and employing (6.10) it follows that $\operatorname{curl} \operatorname{curl} a=0$. The combination of this fact with (6.11) and the identity grad div $-\operatorname{curl}$ curl $=\Delta$ yields

$$
\operatorname{grad} \operatorname{div} a=\Delta a=-\frac{1}{3} \operatorname{grad} \operatorname{div} a
$$

and thus grad $\operatorname{div} a=\Delta a=0$. Since this Poisson's equation is uniquely solvable we conclude $a=0$ and $A=0$, and utilizing (6.9) also $u=$ const. Hence, $u=0$ yielding $T=0$. 


\subsection{There are no DevSym-DevSymCurl- or DevSym-SymCurl-inequalities}

Choose $u \in C_{0}^{\infty}(\Omega ; \mathbb{R})$ and set $T:=u \cdot \mathrm{Id}$. Then $\operatorname{dev} \operatorname{sym} T=0$ and, according to (6.5), $\operatorname{sym} \operatorname{Curl} T=0$. Therefore, such inequalities have to be false.

\subsection{There is no Sym-Div-inequality}

Choose $u \in C_{0}^{\infty}(\Omega ; \mathbb{R})$ and set $a:=\operatorname{grad} u$ and define $A$ according to (6.1). Then we have Div $A=-\operatorname{curl} a=$ -curl $\operatorname{grad} u=0$ and $\operatorname{sym} A=0$. Therefore, such an inequality is false.

\subsection{The DevSymGrad-inequality is false for $n=2$}

As already announced in the introduction, now we show that in the case $n=2$ the trace-free version of Korn's first inequality with only partial boundary condition is false. This is remarkable, since the kernel of the inequality is already controlled by a partial boundary condition. In fact, if a function is in the kernel, then it is holomorphic in $\Omega$. But if a holomorphic function vanishes on some part of the boundary it has to vanish on the whole of $\Omega$. This shows that having a norm on the space under consideration is only necessary for the validity of an inequality. The construction of our counterexample is taken from [30] and in that paper it served as a counterexample to a version of Korn's first inequality with non-constant (rotation) coefficients. For the convenience of the reader we introduce this example in detail, thereby we exactly follow [30].

We identify $\mathbb{R}^{2}$ with $\mathbb{C}$ via standard notation $z=x+i y$. We also use the standard notation for the polar coordinates $(x, y)=r(\cos t, \sin t)$. Consider the sequence

$$
u_{n}(x, y)=x z^{n}
$$

on the half disk $\Omega=\{z:|z|<1, x>0\}$. As $\Gamma_{\tau}$ we choose $\{z \in \partial \Omega: x=0\}$. Then, of course, each of the mappings $u_{n}$ vanishes on $\Gamma_{\tau}$. We first compute $\operatorname{grad} u_{n}(x, y)$. Since

$$
\left(z^{n}\right)^{\prime}=n z^{n-1}=n r^{n-1}(\cos (n t-t)+i \sin (n t-t)),
$$

we obtain

$$
\operatorname{grad} z^{n}=n r^{n-1}\left(\begin{array}{cc}
\cos (n t-t) & -\sin (n t-t) \\
\sin (n t-t) & \cos (n t-t)
\end{array}\right)
$$

Therefore, we have

$$
\begin{aligned}
\operatorname{grad} u_{n}(x, y) & =\left(x \operatorname{grad}_{x}\left(z^{n}\right)+z^{n}, x \operatorname{grad}_{y}\left(z^{n}\right)\right) \\
& =r^{n}\left(\begin{array}{cc}
\cos (n t) & 0 \\
\sin (n t) & 0
\end{array}\right)+n r^{n} \cos t\left(\begin{array}{cc}
\cos (n t-t)-\sin (n t-t) \\
\sin (n t-t) & \cos (n t-t)
\end{array}\right)
\end{aligned}
$$

and hence

$$
\begin{aligned}
\left|\operatorname{grad} u_{n}\right|^{2} & =r^{2 n}+2 n^{2} r^{2 n} \cos ^{2} t+2 r^{2 n} n \cos t(\cos (n t) \cos (n t-t)+\sin (n t) \sin (n t-t)) \\
& =r^{2 n}+2 r^{2 n}\left(n^{2}+n\right) \cos ^{2} t
\end{aligned}
$$

Taking into account that

$$
\int_{-\pi / 2}^{\pi / 2} \cos ^{2} t \mathrm{~d} t=\frac{\pi}{2}
$$

we obtain

$$
\begin{aligned}
\int_{\Omega}\left|\operatorname{grad} u_{n}\right|^{2} & =\int_{0}^{1} \int_{-\pi / 2}^{\pi / 2} r\left(r^{2 n}+2 r^{2 n}\left(n^{2}+n\right) \cos ^{2} t\right) \mathrm{d} t \mathrm{~d} r \\
& =\pi\left(n^{2}+n+1\right) \int_{0}^{1} r^{2 n+1} \mathrm{~d} r=\pi \frac{n^{2}+n+1}{2 n+2}
\end{aligned}
$$


Now, we use this construction as a counterexample for the DevSymGrad-inequality: Switching back to our notation we have

$$
\lim _{n \rightarrow \infty}\left\|\operatorname{Grad} u_{n}\right\|_{L^{2}(\Omega)}=\infty .
$$

On the other hand, inspection of formula (6.12) yields

$$
\begin{aligned}
\operatorname{sym} \operatorname{Grad} u_{n} & =r^{n}\left(\begin{array}{cc}
\cos (n t) & \frac{1}{2} \sin (n t) \\
\frac{1}{2} \sin (n t) & 0
\end{array}\right)+n r^{n} \cos t\left(\begin{array}{cc}
\cos (n t-t) & 0 \\
0 & \cos (n t-t)
\end{array}\right), \\
\operatorname{dev}_{2} \operatorname{sym} \operatorname{Grad} u_{n} & =\frac{1}{2} r^{n}\left(\begin{array}{cc}
\cos (n t) & \sin (n t) \\
\sin (n t) & -\cos (n t)
\end{array}\right),
\end{aligned}
$$

where $\operatorname{dev}_{2} X=X-\frac{1}{2} \operatorname{tr}(X) \cdot$ Id denotes the two-dimensional deviator. Hence,

$$
\left|\operatorname{dev}_{2} \operatorname{sym} \operatorname{Grad} u_{n}\right|^{2}=\frac{1}{2} r^{2 n}
$$

and thus

$$
\left\|\operatorname{dev}_{2} \operatorname{sym} \operatorname{Grad} u_{n}\right\|_{L^{2}(\Omega)}^{2}=\frac{\pi}{4 n+4},
$$

converging to zero in the limit $n \rightarrow \infty$ in contradiction to (6.14) and (1.8).

The fact that the DevSymGrad inequality does not hold in the two-dimensional case is due to the special form of the dev operator in this case. If we instead view the plane symmetric gradient as an object in three dimensions and apply the standard dev operator for $n=3$ (simply denoted by dev in the sequel), then we obtain

$$
\begin{aligned}
\operatorname{dev} \operatorname{sym} \operatorname{Grad} u_{n}= & r^{n}\left(\begin{array}{ccc}
\frac{2}{3} \cos (n t) & \frac{1}{2} \sin (n t) & 0 \\
\frac{1}{2} \sin (n t) & -\frac{1}{3} \cos (n t) & 0 \\
0 & 0 & -\frac{1}{3} \cos (n t)
\end{array}\right) \\
& +n r^{n} \cos t \cos (n t-t)\left(\begin{array}{ccc}
\frac{1}{3} & 0 & 0 \\
0 & \frac{1}{3} & 0 \\
0 & 0 & -\frac{2}{3}
\end{array}\right)
\end{aligned}
$$

This implies

$$
\begin{aligned}
\left|\operatorname{dev} \operatorname{sym} \operatorname{Grad} u_{n}\right|^{2} & =r^{2 n}\left(\frac{2}{3} \cos ^{2}(n t)+\frac{1}{2} \sin ^{2}(n t)\right)+\frac{2}{3} n^{2} r^{2 n} \cos ^{2} t \cos ^{2}(n t-t) \\
& \geq \frac{2}{3} n^{2} r^{2 n} \cos ^{2} t \cos ^{2}(n t-t)
\end{aligned}
$$

and, for $n>2$,

$$
\left\|\operatorname{dev}^{3 \mathrm{D}} \operatorname{sym} \operatorname{Grad} u_{n}\right\|_{L^{2}(\Omega)}^{2} \geq \frac{2}{3} n^{2} \frac{\pi}{4} \frac{1}{2 n+2}=\frac{\pi n^{2}}{12(n+1)},
$$

where we used the fact that

$$
\int_{-\pi / 2}^{\pi / 2} \cos ^{2} t \cos ^{2}(n t-t) \mathrm{d} t=\frac{\pi}{4}
$$

holds for $n>2$. This means that $\left\|\operatorname{dev} \operatorname{sym} \operatorname{Grad} u_{n}\right\|_{L^{2}(\Omega)}^{2} \rightarrow \infty$ for $n \rightarrow \infty$ in concordance with Lemma 4.2.

\section{Applichtions}

In this section we will present some prototype applications where the new inequalities may be used to establish coercivity of the models. 


\subsection{Infinitesimal incompressible elasticity}

Historically, inequalities like the one in Theorem 3.1 first appeared in the context of mixed stress-displacement formulations of linear elasticity in the incompressible limit ( $c f .[2])$. The result in [2] is stated in two dimensions assuming vanishing average trace (see also [3], Prop. 9.1.1). It is generalized in [9] using a different argument assuming only that the identity tensor is eliminated by some constraint. In the incompressible limit, the mixed variational formulation of linear elasticity turns into the problem of finding some $\sigma \in H\left(\operatorname{Div} ; \Gamma_{\nu} ; \Omega\right), u \in$ $L^{2}\left(\Omega ; \mathbb{R}^{n}\right)$ and $\gamma \in L^{2}(\Omega ; \mathfrak{s o}(n))$, such that

$$
\begin{aligned}
(\operatorname{dev} \sigma, \tau)_{L^{2}(\Omega)}+(u, \operatorname{Div} \tau)_{L^{2}(\Omega)}+(\gamma, \text { skew } \tau)_{L^{2}(\Omega)} & =0 \\
(\operatorname{Div} \sigma, v)_{L^{2}(\Omega)}+(f, v)_{L^{2}(\Omega)} & =0, \\
(\text { skew } \sigma, \eta)_{L^{2}(\Omega)} & =0
\end{aligned}
$$

holds for all $\tau \in H\left(\operatorname{Div} ; \Gamma_{\nu} ; \Omega\right), v \in L^{2}\left(\Omega ; \mathbb{R}^{n}\right)$ and $\eta \in L^{2}(\Omega ; \mathfrak{s o}(n))$. This saddle-point problem may be viewed as the Karush-Kuhn-Tucker system associated with minimizing the elastic energy with respect to the stresses subject to momentum balance and symmetry as constraints. Its well-posedness relies on the estimate in Theorem 3.1. The same is true for the stress-displacement first-order system least squares approach studied in $[5]$.

\subsection{Pseudostress formulation of stationary Stokes equations}

Here, the following formulation of the stationary Stokes equations is considered: For some given $f: \Omega \rightarrow \mathbb{R}^{3}$ find the pressure $p: \Omega \rightarrow \mathbb{R}$, the velocity $u: \Omega \rightarrow \mathbb{R}^{3}$ and the stress $\sigma: \Omega \rightarrow \mathbb{R}^{3 \times 3}$ such that the first-order system

$$
\sigma-\mu \operatorname{sym} \operatorname{grad} u+p \operatorname{Id}=0, \quad \operatorname{Div} \sigma=f, \quad \operatorname{div} u=0
$$

holds in $\Omega$. This system is obviously equivalent to

$$
\operatorname{dev} \sigma-\mu \operatorname{sym} \operatorname{grad} u=0, \quad \operatorname{Div} \sigma=f,
$$

where the pressure $p$ has been eliminated and can be computed afterwards as $p=-\operatorname{tr}(\sigma) / 3$. For this first-order system, a least squares formulation based on minimizing the quadratic functional

$$
\|\operatorname{dev} \sigma-\mu \operatorname{sym} \operatorname{grad} u\|_{L^{2}(\Omega)}^{2}+\|\operatorname{Div} \sigma-f\|_{L^{2}(\Omega)}^{2}
$$

with respect to $u$ and $\sigma$ may be used. In order to obtain a coercivity result for this functional, let us first investigate the mixed terms arising in the first part of the functional, leading to

$$
\begin{aligned}
(\operatorname{dev} \sigma, \operatorname{sym} \operatorname{grad} u)_{L^{2}(\Omega)} & =(\operatorname{sym} \operatorname{dev} \sigma, \operatorname{grad} u)_{L^{2}(\Omega)}=\left(\operatorname{sym} \sigma-\frac{1}{3} \operatorname{tr}(\sigma) \operatorname{Id}, \operatorname{grad} u\right)_{L^{2}(\Omega)} \\
& =(\sigma, \operatorname{grad} u)_{L^{2}(\Omega)}-(\operatorname{skew} \sigma, \operatorname{grad} u)_{L^{2}(\Omega)}-\frac{1}{3}(\operatorname{tr}(\sigma), \operatorname{div} u)_{L^{2}(\Omega)} \\
& =-(\operatorname{Div} \sigma, u)_{L^{2}(\Omega)}-(\operatorname{skew} \sigma, \operatorname{grad} u)_{L^{2}(\Omega)}-\frac{1}{3}(\operatorname{tr}(\sigma), \operatorname{div} u)_{L^{2}(\Omega)},
\end{aligned}
$$

if we assume proper boundary conditions on $\sigma$ and $u$, justifying the partial integration without boundary terms, i.e., $\sigma \in H\left(\operatorname{Div} ; \Gamma_{\nu} ; \Omega\right)$ and $u \in H\left(\operatorname{Grad} ; \Gamma_{\tau} ; \Omega\right)$. This implies, for arbitrary $\delta \in(0,1)$,

$$
\begin{aligned}
2 \mu(\operatorname{dev} \sigma, \operatorname{sym} \operatorname{grad} u)_{L^{2}(\Omega)} \leq & \delta\left(\mu^{2}\|u\|_{L^{2}(\Omega)}^{2}+\mu^{2}\|\operatorname{grad} u\|_{L^{2}(\Omega)}^{2}+\frac{1}{3}\|\operatorname{tr}(\sigma)\|_{L^{2}(\Omega)}^{2}\right) \\
& +\frac{1}{\delta}\left(\|\operatorname{Div} \sigma\|_{L^{2}(\Omega)}^{2}+\|\operatorname{skew} \sigma\|_{L^{2}(\Omega)}^{2}+\frac{1}{3} \mu^{2}\|\operatorname{div} u\|_{L^{2}(\Omega)}^{2}\right) .
\end{aligned}
$$


If we combine this with the straightforward estimates

$$
\begin{aligned}
\|\operatorname{skew} \sigma\|_{L^{2}(\Omega)} & =\|\operatorname{skew}(\operatorname{dev} \sigma-\mu \operatorname{sym} \operatorname{grad} u)\|_{L^{2}(\Omega)} \leq\|\operatorname{dev} \sigma-\mu \operatorname{sym} \operatorname{grad} u\|_{L^{2}(\Omega)}, \\
\mu\|\operatorname{div} u\|_{L^{2}(\Omega)} & =\|\operatorname{tr}(\operatorname{dev} \sigma-\mu \operatorname{sym} \operatorname{grad} u)\|_{L^{2}(\Omega)} \leq \sqrt{3}\|\operatorname{dev} \sigma-\mu \operatorname{sym} \operatorname{grad} u\|_{L^{2}(\Omega)},
\end{aligned}
$$

we are led to

$$
\begin{aligned}
\|\operatorname{dev} \sigma-\mu \operatorname{sym} \operatorname{grad} u\|_{L^{2}(\Omega)}^{2}+\|\operatorname{Div} \sigma\|_{L^{2}(\Omega)}^{2} \\
\geq \\
\geq \frac{1}{3}\left(\|\operatorname{dev} \sigma-\mu \operatorname{sym} \operatorname{grad} u\|_{L^{2}(\Omega)}^{2}+\|\operatorname{skew} \sigma\|_{L^{2}(\Omega)}^{2}+\frac{\mu^{2}}{3}\|\operatorname{div} u\|_{L^{2}(\Omega)}^{2}+\|\operatorname{Div} \sigma\|_{L^{2}(\Omega)}^{2}\right) \\
\geq \frac{\delta}{6}\left(\|\operatorname{dev} \sigma-\mu \operatorname{sym} \operatorname{grad} u\|_{L^{2}(\Omega)}^{2}+\frac{2}{\delta}\left(\|\operatorname{skew} \sigma\|_{L^{2}(\Omega)}^{2}+\frac{\mu^{2}}{3}\|\operatorname{div} u\|_{L^{2}(\Omega)}^{2}+\|\operatorname{Div} \sigma\|_{L^{2}(\Omega)}^{2}\right)\right) \\
\geq \frac{\delta}{6}\left(\|\operatorname{dev} \sigma\|_{L^{2}(\Omega)}^{2}+\mu^{2}\|\operatorname{sym} \operatorname{grad} u\|_{L^{2}(\Omega)}^{2}-\delta\left(\mu^{2}\|u\|_{L^{2}(\Omega)}^{2}+\mu^{2}\|\operatorname{grad} u\|_{L^{2}(\Omega)}^{2}+\frac{1}{3}\|\operatorname{tr}(\sigma)\|_{L^{2}(\Omega)}^{2}\right)\right. \\
\left.\quad+\frac{1}{\delta}\left(\|\operatorname{Div} \sigma\|_{L^{2}(\Omega)}^{2}+\|\operatorname{skew} \sigma\|_{L^{2}(\Omega)}^{2}+\frac{1}{3} \mu^{2}\|\operatorname{div} u\|_{L^{2}(\Omega)}^{2}\right)\right) \\
\geq \frac{\delta}{6}\left(\|\operatorname{dev} \sigma\|_{L^{2}(\Omega)}^{2}+\|\operatorname{Div} \sigma\|_{L^{2}(\Omega)}^{2}+\mu^{2}\|\operatorname{sym} \operatorname{grad} u\|_{L^{2}(\Omega)}^{2}-\delta \mu^{2}\|u\|_{H(\operatorname{Grad} ; \Omega)}^{2}-\frac{\delta}{3}\|\operatorname{tr}(\sigma)\|_{L^{2}(\Omega)}^{2}\right) \\
\geq \frac{\delta}{6}\left(\frac{1}{C_{D D}^{2}}\|\sigma\|_{H(\operatorname{Div} ; \Omega)}^{2}+\frac{\mu^{2}}{C_{K P}^{2}}\|u\|_{H(\operatorname{Grad} ; \Omega)}^{2}-\delta \mu^{2}\|u\|_{H(\operatorname{Grad} ; \Omega)}^{2}-\delta\|\sigma\|_{L^{2}(\Omega)}^{2}\right)
\end{aligned}
$$

for all $\delta \leq 1$ with $C_{D D}$ from Theorem 3.1 and the Korn-Poincaré constant $C_{K P}$ in the Korn-Poincaré's inequality

$$
C_{K P}\|\operatorname{sym} \operatorname{grad} u\|_{L^{2}(\Omega)} \geq C_{K}\|\operatorname{grad} u\|_{L^{2}(\Omega)} \geq\|u\|_{H(\operatorname{Grad} ; \Omega)} .
$$

Choosing $\delta$ sufficiently small gives us the desired coercivity estimate

$$
\|\operatorname{dev} \sigma-\mu \operatorname{sym} \operatorname{grad} u\|_{L^{2}(\Omega)}^{2}+\|\operatorname{Div} \sigma\|_{L^{2}(\Omega)}^{2} \geq C\left(\|\operatorname{Div} \sigma\|_{H(\operatorname{Div} ; \Omega)}^{2}+\|u\|_{H(\operatorname{Grad} ; \Omega)}^{2}\right) .
$$

The pseudostress-velocity formulation of the stationary Stokes's equations introduced above was studied in ([7], Sect. 3.2) (see also $[8,15]$ for related mixed finite element approaches). It was used as a basis for the treatment of Stokes-Darcy interface problems by a first-order system least squares approach in [19]. Recently, a pseudostress-based approach for the stationary Navier-Stokes was investigated in [6].

\subsection{Pseudostress formulation of generalized Newtonian flow}

The estimate of Theorem 3.1 is also useful in the context of nonlinear generalized Newtonian fluids which differs from the formulation above in that the viscosity may depend on the velocity gradient $\mu=\mu(\operatorname{grad} u)$. Very popular is Carreau's law, where this nonlinear dependence is given by

$$
\mu(\operatorname{grad} u)=\mu_{0}\left(1+|\operatorname{sym} \operatorname{grad} u|^{2}\right)^{(r-2) / 2}
$$

with $\mu_{0}>0$ and $r \geq 1$. Depending on the value of $r$, shear-thickening or shear-thinning behavior of the fluid can be modeled. A dual-mixed approach to nonlinear generalized Newtonian Stokes flow was introduced and analyzed in [12]. This model may also be treated by a pseudostress-velocity approach in a first-order system least squares setting based on minimizing the nonlinear functional

$$
\|\operatorname{dev} \sigma-\mu(\operatorname{grad} u) \operatorname{sym} \operatorname{grad} u\|_{L^{2}(\Omega)}^{2}+\|\operatorname{Div} \sigma-f\|_{L^{2}(\Omega)}^{2} .
$$

Such a method is studied in detail in [18]. 


\subsection{Infinitesimal gradient plasticity}

Phenomenological plasticity models are intended to describe the irreversible deformation behavior of metals. There exists a great variety of models. Here we focus on rate-dependent or rate-independent models with kinematic hardening. The system of equations consist of balance of linear momentum coupled with a local nonlinear evolution equation in each space point for the plastic variable.

In many new applications, the size of the considered specimen is so small, that size effects need to be taken into account. Instead of a local evolution problem we have to consider a nonlinear evolution problem where the right hand side contains certain combinations of second partial derivatives of the plastic distortion.

For the setting of the nonlinear gradient-plasticity problem, let $\Omega \subset \mathbb{R}^{3}$ be an open and bounded set, the set of material points of the solid body. By $T_{e}$ we denote a positive number (time of existence). Unknown in our small strain formulation are the displacement field $u: \Omega \times\left[0, T_{e}\right) \rightarrow \mathbb{R}^{3}$ of the material point $x$ at time $t$ and the non-symmetric infinitesimal plastic distortion $P: \Omega \times\left[0, T_{e}\right) \rightarrow \mathfrak{s l}(3)$. The model equations of the problem are

$$
\begin{aligned}
\operatorname{Div} \sigma & =f, \\
\sigma & =2 \mu(\operatorname{sym}(\operatorname{grad} u-P))+\lambda \operatorname{tr}(\operatorname{grad} u) \cdot \mathrm{Id}, \\
\partial_{t} P(x, t) & \in g\left(x, \Sigma^{\operatorname{lin}}(x, t)\right), \\
\Sigma^{\operatorname{lin}} & =\Sigma_{\mathrm{e}}^{\operatorname{lin}}+\Sigma_{\mathrm{sh}}^{\operatorname{lin}}+\Sigma_{\mathrm{curl}}^{\operatorname{lin}}, \\
\Sigma_{\mathrm{e}}^{\operatorname{lin}} & =\sigma, \quad \Sigma_{\mathrm{sh}}^{\operatorname{lin}}=-\operatorname{dev} \operatorname{sym} P, \quad \Sigma_{\text {curl }}^{\operatorname{lin}}=-\operatorname{Curl} \operatorname{Curl} P,
\end{aligned}
$$

which must be satisfied in $\Omega \times\left[0, T_{e}\right)$. Here, $\Sigma^{\text {lin }}$ is the infinitesimal Eshelby stress tensor driving the evolution of the plastic distortion $P$. The initial and boundary conditions are

$$
\begin{aligned}
P(\cdot, 0) & =P_{0} & & \text { in } \Omega, \\
\nu \times P & =0 & & \text { on } \partial \Omega \times\left[0, T_{e}\right), \\
u & =0 & & \text { on } \partial \Omega \times\left[0, T_{e}\right),
\end{aligned}
$$

where $\nu$ is a normal vector on the boundary $\partial \Omega$. For the model we require that the nonlinear constitutive mapping $(\Sigma \rightarrow g(\cdot, \Sigma)): \mathbb{R}^{3 \times 3} \rightarrow 2^{\mathfrak{s l}(3)}$ is monotone. Given are the volume force $f: \Omega \times\left[0, T_{e}\right) \rightarrow \mathbb{R}^{3}$ and the initial datum $P_{0}: \Omega \rightarrow \mathfrak{s l}(3)$. It is easy to see that the corresponding free energy of the system is

$$
\mu\|\operatorname{sym}(\operatorname{grad} u-P)\|_{L^{2}(\Omega)}^{2}+\frac{\lambda}{2}\|\operatorname{tr}(\operatorname{grad} u)\|_{L^{2}(\Omega)}^{2}+\frac{1}{2}\|\operatorname{dev} \operatorname{sym} P\|_{L^{2}(\Omega)}^{2}+\frac{1}{2}\|\operatorname{Curl} P\|_{L^{2}(\Omega)}^{2} .
$$

The appearance of Curl $P$ instead of the full gradient $\operatorname{grad} P$ is dictated by dislocation mechanics, the appearance of dev sym $P$ instead of $P$ is dictated by invariance of the model under superposition of infinitesimal rotations. Here, coercivcity is obtained by using the DevSym-Curl inequality. Model equations similar to the above problem have been considered in [25].

\subsection{Infinitesimal Cosserat elasticity}

Cosserat or micropolar elasticity is intended to describe materials with a microstructure which has the degrees of freedom of a rigid body. With Cosserat elasticity, it is possible to describe some form of elastic size effects (smaller samples are comparatively stiffer) and wave dispersion in the case of dynamic equations. Here, we consider the static problem. In a variational context, the problem is completely described by writing the energy which is to be minimized. We are looking for the displacement $u: \Omega \rightarrow \mathbb{R}^{3}$ and the infinitesimal Cosserat microrotation $A: \Omega \rightarrow \mathfrak{s o}(3)$ minimizing the two-field functional

$$
\begin{gathered}
\mu\|\operatorname{sym} \operatorname{grad} u\|_{L^{2}(\Omega)}^{2}+\mu_{c}\|\operatorname{skew}(\operatorname{grad} u-A)\|_{L^{2}(\Omega)}^{2}+\frac{\lambda}{2}\|\operatorname{tr}(\operatorname{grad} u)\|_{L^{2}(\Omega)}^{2} \\
+\frac{1}{2}\|\operatorname{dev} \operatorname{sym} \operatorname{Curl} A\|_{L^{2}(\Omega)}^{2}+(f, u)_{L^{2}(\Omega)} .
\end{gathered}
$$


The corresponding system of Euler-Lagrange's equations in strong form are

$$
\begin{aligned}
\operatorname{Div} \sigma & =f, \\
\sigma & =2 \mu \operatorname{sym} \operatorname{grad} u+\lambda \operatorname{tr}(\operatorname{grad} u) \cdot \operatorname{Id}+2 \mu_{c} \operatorname{skew}(\operatorname{grad} u-A), \\
\text { skew } \sigma & =\operatorname{skew} \text { Curl dev sym } \operatorname{Curl} A .
\end{aligned}
$$

The form of the curvature contribution $\operatorname{dev} \operatorname{sym} \operatorname{Curl} A$ instead of the full gradient $\operatorname{grad} A$ is motivated by conformal invariance of the model, see [23]. Here, a variant of the DevSym-DevCurl inequality is applicable. Model equations similar to the above problem have been considered in [23].

\subsection{Infinitesimal Cosserat elasto-plasticity}

Frequently encountered are also couplings between Cosserat elasticity and plasticity models. However, plasticity in these models is treated classically as a local phenomenon. We are looking for the displacement $u: \Omega \times\left[0, T_{e}\right) \rightarrow \mathbb{R}^{3}$, the infinitesimal Cosserat micro-rotation $A: \Omega \times\left[0, T_{e}\right) \rightarrow \mathfrak{s o}(3)$ and the plastic distortion $P: \Omega \times\left[0, T_{e}\right) \rightarrow \mathfrak{s l}(3)$ satisfying

$$
\begin{aligned}
\operatorname{Div} \sigma & =f, \\
\sigma & =2 \mu(\operatorname{sym}(\operatorname{grad} u-P))+\lambda \operatorname{tr}(\operatorname{grad} u) \cdot \operatorname{Id}+2 \mu_{c} \operatorname{skew}(\operatorname{grad} u-A), \\
\partial_{t} P(x, t) & \in g(x, \operatorname{sym} \sigma(x, t)), \\
\text { skew } \sigma & =\text { skew } \operatorname{Curl} \operatorname{dev} \operatorname{Curl} A .
\end{aligned}
$$

Model equations with these features have been considered in $[21,24]$ with the purpose of obtaining regularizations of classical plasticity models.

\subsection{Infinitesimal relaxed micromorphic elasticity}

Micromorphic extended continuum models assume that at each material point there is a microstructure attached which itself may deform as an elastic body. In a variational context, the problem is completely described by writing down the energy which is to be minimized. We are looking for the displacement fields $u: \Omega \rightarrow \mathbb{R}^{3}$ and the not necessarily symmetric micromorphic distortion $P: \Omega \rightarrow \mathbb{R}^{3 \times 3}$ minimizing

$$
\begin{aligned}
& \mu\|\operatorname{sym}(\operatorname{grad} u-P)\|_{L^{2}(\Omega)}^{2}+\frac{\lambda}{2}\|\operatorname{tr}(\operatorname{grad} u-P)\|_{L^{2}(\Omega)}^{2} \\
& \quad+\frac{1}{2}\|\operatorname{dev} \operatorname{sym} P\|_{L^{2}(\Omega)}^{2}+\frac{1}{2}\|\operatorname{Curl} P\|_{L^{2}(\Omega)}^{2}+(f, u)_{L^{2}(\Omega)} .
\end{aligned}
$$

The corresponding system of Euler-Lagrange's equations in strong form are

$$
\begin{aligned}
\operatorname{Div} \sigma & =f, \\
\sigma & =2 \mu \operatorname{sym}(\operatorname{grad} u-P)+\lambda \operatorname{tr}(\operatorname{grad} u-P) \cdot \operatorname{Id}, \\
\text { Curl dev } \operatorname{Curl} P & =-\operatorname{dev} \operatorname{sym} P+\sigma .
\end{aligned}
$$

An important feature, which sets this model apart from more classical micromorphic approaches, is that the balance of forces does not 'see' derivatives of $P$ since Div Curl $=0$. Here, the DevSym-DevCurl-inequality is applicable. Model equations similar to the above problem have been considered in [28].

\section{Appendix A. The KeRnel of Dev Sym Grad}

For the convenience of the reader we compute the representation formulae (4.6) and (4.8) of vector fields in the kernel of dev sym Grad, used in the proof of Lemma 4.2. These mappings are often called conformal 
mappings or conformal Killing vectors. Such a representation is given, e.g., in [32]. Now let $\Omega \subset \mathbb{R}^{n}$ be an arbitrary domain, $n \geq 3$ and $v \in H(\operatorname{Grad} ; \Omega)$ with dev $\operatorname{sym} \operatorname{Grad} v=0$. Then

$$
\operatorname{Grad} v=u \cdot \operatorname{Id}+A,
$$

where after selecting a suitable representant $u(x) \in \mathbb{R}$ and $A(x)$ is a skew-symmetric $(n \times n)$-matrix for all $x \in \Omega$. Since Curl Grad $v=0$ we obtain that for all $i, j, k=1, \ldots, n$

$$
\left(\partial_{j} u\right) \delta_{i k}-\left(\partial_{k} u\right) \delta_{i j}=-\partial_{j} A_{i k}+\partial_{k} A_{i j} .
$$

Now assume that $i, j, k$ are pairwise different, then using (A.2) and $A_{i j}=-A_{j i}$ we compute

$$
\partial_{j} A_{i k}=\partial_{k} A_{i j}=-\partial_{k} A_{j i}=-\partial_{i} A_{j k}=\partial_{i} A_{k j}=\partial_{j} A_{k i}=-\partial_{j} A_{i k},
$$

yielding $\partial_{j} A_{i k}=0$. Now assume $j=i$, but $k \neq i$. In this case we obtain by (A.2)

$$
\partial_{j} A_{j k}=\partial_{k} u \text {. }
$$

Therefore,

$$
\begin{array}{ll}
\partial_{j} A_{j k}=\partial_{k} u & \text { if } \quad j \neq k, \\
\partial_{j} A_{k j}=-\partial_{k} u & \text { if } \quad j \neq k, \\
\partial_{j} A_{i k}=0 & \text { if } \quad i \neq j, i \neq k, j \neq k, \\
\partial_{j} A_{i i}=0 . &
\end{array}
$$

Now we show that (A.3) implies $\operatorname{grad} u=$ const. First assume $j \neq k$ and choose $i$ with $i \neq j$ and $i \neq k$ (since $n \geq 3$, this is possible). Therefore we obtain

$$
\partial_{k} \partial_{j} u=\partial_{k} \partial_{i} A_{i j}=\partial_{i} \partial_{k} A_{i j}=0 .
$$

Now, we assume that $i \neq j$, then

$$
\partial_{j} \partial_{j} u=\partial_{j} \partial_{i} A_{i j}=-\partial_{j} \partial_{i} A_{j i}=-\partial_{i} \partial_{j} A_{j i}=-\partial_{i} \partial_{i} u .
$$

As $n \geq 3$ we can play the indices against each other and obtain

$$
\partial_{j} \partial_{j} u=-\partial_{i} \partial_{i} u=\partial_{k} \partial_{k} u=-\partial_{j} \partial_{j} u=0
$$

for $i, j, k$ pairwise different. Therefore $\operatorname{grad} u=$ const. $=\bar{w} \in \mathbb{R}^{n}$ and after a possible redefinition on a set of measure zero, we get

$$
u(x)=\bar{w} \cdot x+\bar{u}
$$

with $\bar{u} \in \mathbb{R}$. Note $\partial_{i} u=\bar{w}_{i}$. Since $\partial_{k} A_{i j}$ is constant, see (A.3), we also know that

$$
A_{i j}(x)=\sum_{k=1}^{n} \partial_{k} A_{i j} x_{k}+\bar{A}_{i j}=\sum_{k=1}^{n} \bar{a}_{i j k} x_{k}+\bar{A}_{i j}=\bar{w}_{j} x_{i}-\bar{w}_{i} x_{j}+\bar{A}_{i j}
$$

with $\bar{a}_{i j k}$ from (4.9) and some skew-symmetric constant matrix $\bar{A}$. Utilizing (A.1), (A.4) and (A.5) we conclude that (4.7) holds true. Furthermore, by integrating the $i$-th component of $v$ we obtain from (A.1), i.e.,

$$
\partial_{j} v_{i}(x)=u(x) \delta_{i j}+A_{i j}(x)=u(x) \delta_{i j}+\bar{w}_{j} x_{i}-\bar{w}_{i} x_{j}+\bar{A}_{i j},
$$

immediately

$$
v_{i}(x)=u(x) x_{i}-\frac{1}{2} \bar{w}_{i}|x|^{2}+\bar{A}_{i k} x_{k}+\bar{v}_{i}
$$

or as a vector

$$
v(x)=u(x) x-\frac{1}{2}|x|^{2} \bar{w}+\bar{A} x+\bar{v}
$$

which is (4.6).

Acknowledgements. We heartily thank Kostas Pamfilos for his beautiful pictures of 3D sliceable domains. 


\section{REFERENCES}

[1] R.A. Adams, Sobolev Spaces. Academic Press, New York (1975).

[2] D.N. Arnold, J. Douglas and C.P. Gupta, A family of higher order mixed finite element methods for plane elasticity. Numer. Math. 45 (1984) 1-22.

[3] D. Boffi, F. Brezzi and M. Fortin, Mixed Finite Element Methods and Applications. Springer, Heidelberg (2013).

[4] Z. Cai and G. Starke, First-order system least squares for the stress-displacement formulation: Linear elasticity. SIAM J. Numer. Anal. 41 (2003) 715-730.

[5] Z. Cai and G. Starke, Least squares methods for linear elasticity. SIAM J. Numer. Anal. 42 (2004) 826-842.

[6] Z. Cai and S. Zhang, Mixed methods for stationary Navier-Stokes equations based on pseudostress-pressure-velocity formulation. Math. Comput. 81 (2012) 1903-1927.

[7] Z. Cai, B. Lee and P. Wang, Least squares methods for incompressible Newtonian fluid flow: Linear stationary problems. SIAM J. Numer. Anal. 42 (2004) 843-859.

[8] Z. Cai, C. Tong, P.S. Vassilevski and C. Wang, Mixed finite element methods for incompressible flow: Stationary Stokes equations. Numer. Methods Partial Differ. Equ. 26 (2010) 957-978.

[9] C. Carstensen and G. Dolzmann, A posteriori error estimates for mixed FEM in elasticity. Numer. Math. 81 (1998) 187-209.

[10] P.G. Ciarlet, On Korn's inequality. Chin. Ann. Math. B 31 (2010) 607-618.

[11] S. Dain, Generalized Korn's inequality and conformal Killing vectors. Calc. Var. Partial Differ. Equ. 25 (2006) 535-540.

[12] V.J. Ervin, J.S. Howell and I. Stanculescu, A dual-mixed approximation method for a three-field model of a nonlinear generalized Stokes problem. Comput. Methods Appl. Mech. Engrg. 197 (2008) 2886-2900.

[13] M. Fuchs and O. Schirra, An application of a new coercive inequality to variational problems studied in general relativity and in Cosserat elasticity giving the smoothness of minimizers. Arch. Math. (Basel) 93 (2009) 587-596.

[14] M. Fuchs and S. Repin, Some Poincaré-type inequalities for functions of bounded deformation involving the deviatoric part of the symmetric gradient. Zap. Nauchn. sem. St.-Petersburg Odtel. Math. Inst. Steklov (POMI) 385 (2010) $224-234$.

[15] G. Gatica, A. Márquez and M.A. Sánchez, Analysis of a velocity-pressure-pseudostress formulation for the stationary Stokes equations. Comput. Methods Appl. Mech. Engrg. 199 (2010) 1064-1079.

[16] T. Jakab, I. Mitrea and M. Mitrea, On the regularity of differential forms satisfying mixed boundary conditions in a class of Lipschitz domains. Indiana Univ. Math. J. 58 (2009) 2043-2071.

[17] F. Jochmann, A compactness result for vector fields with divergence and curl in $L^{q}(\Omega)$ involving mixed boundary conditions. Appl. Anal. 66 (1997) 189-203.

[18] S. Münzenmaier, First-order system least squares for generalized-Newtonian coupled Stokes-Darcy flow. Numer. Methods Partial Differ. Equ. 31 (2015) 1150-1173.

[19] S. Münzenmaier and G. Starke, First-order system least squares for coupled Stokes-Darcy flow. SIAM J. Numer. Anal. 49 (2011) 387-404.

[20] J. Nečas, Sur les normes équivalentes dans $W_{p}^{k}(\Omega)$ et sur la coercivité des formes formellement positives, in Équations aux derivées partielles. Les Presses de l'Université de Montréal (1967) 102-128.

[21] P. Neff and K. Chełmiński, Infinitesimal elastic-plastic Cosserat micropolar theory. Modelling and global existence in the rate independent case. Proc. Roy. Soc. Edinb. A 135 (2005) 1017-1039.

[22] P. Neff and I. Münch, Curl bounds Grad on SO(3). ESAIM: COCV 14 (2008) 148-159.

[23] P. Neff and J. Jeong, A new paradigm: the linear isotropic Cosserat model with conformally invariant curvature energy. $Z$. Angew. Math. Mech. 89 (2009) 107-122.

[24] P. Neff, K. Chełmiński, W. Müller and C. Wieners, A numerical solution method for an infinitesimal elastic-plastic Cosserat model. M3AS Math. Mod. Meth. Appl. Sci. 17 (2007) 1211-1239.

[25] P. Neff, K. Chełmiński and H.D. Alber, Notes on strain gradient plasticity. Finite strain covariant modelling and global existence in the infinitesimal rate-independent case. M3AS Math. Mod. Meth. Appl. Sci. 19 (2009) 1-40.

[26] P. Neff, D. Pauly and K.J. Witsch, On a canonical extension of Korn's first inequality to H(Curl) motivated by gradient plasticity with plastic spin. C. R. Math. 349 (2011) 1251-1254.

[27] P. Neff, D. Pauly and K.J. Witsch, Maxwell meets Korn: a new coercive inequality for tensor fields in $\mathbb{R}^{N \times N}$ with squareintegrable exterior derivative. Math. Methods Appl. Sci. 35 (2012) 65-71.

[28] P. Neff, I.D. Ghiba, A. Madeo, L. Placidi and G. Rosi, A unifying perspective: the relaxed linear micromorphic continuum. Cont. Mech. Thermodyn. 26 (2014) 639-681.

[29] P. Neff, D. Pauly and K.J. Witsch, Poincaré meets Korn via Maxwell: Extending Korn's first inequality to incompatible tensor fields. J. Differ. Equ. 258 (2015) 1267-1302.

[30] W. Pompe, Counterexamples to Korn's inequality with non-constant rotation coefficients. Math. Mech. Solids 16 (2011) $172-176$.

[31] Y.G. Reshetnyak, Stability Theorems in Geometry and Analysis. Kluwer Academic Publishers, London (1994).

[32] O. Schirra, New Korn-type inequalities and regularity of solutions to linear elliptic systems and anisotropic variational problems involving the trace-free part of the symmetric gradient. Calc. Var. Partial Differ. Equ. 43 (2012) 147-172.

[33] H. Sohr, The Navier-Stokes Equations. Birkhäuser, Basel (2001).

[34] K. Yoshida, Functional Analysis. Springer-Verlag, Berlin, 6th edition (1980). 